\title{
A realistic transport model with pressure dependent parameters for gas flow in tight porous media with application to determining shale rock properties
}

\author{
Iftikhar Ali and Nadeem A. Malik ${ }^{1}$ \\ Department of Mathematics and Statistics, King Fahd University of Petroleum and \\ Minerals, P.O. Box 5046, Dhahran 31261, Saudi Arabia.
}

\begin{abstract}
Shale gas recovery has seen a major boom in recent years due to the increasing global energy demands; but the extraction technologies are very expensive. It is therefore important to develop realistic transport modelling and simulation methods, for porous rocks and porous media, that can compliment the field work. Here, a new nonlinear transport model for single phase gas flow in tight porous media is derived, incorporating many important physical processes that occur in such porous systems: continuous flow, transition flow, slip flow, Knudsen diffusion, adsorption and desorption in to and out of the rock material, and a correction for high flow rates (turbulence). This produces a nonlinear advection-diffusion type of partial differential equation (PDE) with pressure dependent model parameters and associated compressibility coefficients, and highly nonlinear apparent convective flux (velocity) and apparent diffusivity. An important application is to the determination of shale rock properties, such as porosity and permeability, by history matching of the the simulation results to data from pressure-pulse decay tests in a shale rock core sample [Pong K., Ho C., Liu J., Tai Y. Non-linear pressure distribution in uniform microchannels. ASME Fluids Eng. Div. (FED) Vol. 197, 51-56, (1994)]. The estimates of the rock porosity and the permeability from our model simulations are realistic of shale rocks, more realistic than obtained from previous models, and illustrates
\end{abstract}

\footnotetext{
${ }^{1}$ Corresponding: namalik@kfupm.edu.sa and nadeem_malik@cantab.net
} 
the potential of the modelling strategy presented here in producing accurate simulations of shale gas flow in tight reservoirs.

Keywords: Shale, gas, transport, porous media, tight, rocks, pressure, porosity, permeability, modelling and simulations.

\section{Introduction: Global Energy Perspective and Shale Gas}

At the current time, global energy demand is met by a number of energy resources that includes oil, gas, coal, biomass, nuclear, solar, hydro, wind and other renewable energy sources. Hydrocarbon based fuels meet more than $80 \%$ of the world's energy requirements and the world energy supply will continue to depend heavily on carbon based fuels because they are abundant and inexpensive. In order to provide an uninterrupted supply of hydrocarbon fuels and to meet the future energy demands, new avenues are being explored, both conventional and unconventional, Wang et al. 83] and Islam [43]. Among the latter, shale gas recovery has attracted a lot of attention in the oil and gas industry recently, Soeder [74] and Arthur et al. [5].

Shales are sedimentary rocks that are found abundantly (40\%) on earth. The rock structure in shale reservoirs is complex, possessing micropores, nanpores, and also microcracks. Fluid flow in porous media occurs in interconnected networks of void spaces, see Figure 1. In general, the fluid may be of single phase (either liquid or gas), or two phase (both liquid and gas), or even multiphase because solid particles can also be transported within the pores. Furthermore, the fluid may contain several chemical species, sometimes deliberately added, such as methanol, lead, sulfuric acid, silica, water. Solvents are used to open up channels and pore spaces, and coagulants are added to block them. New advanced technologies such as hydraulic fracturing and horizontal drilling are critical for the extraction of the natural gas, Estrada and Bhamidimarri [29. These are very expensive techniques, so they are often augmented by transport 
modelling and simulation methods which may indicate future flow rates, and may also be used to estimate rock properties, Ma et al. [51.

Shales have very small pore size compared to conventional rock formations. Different ranges of pore size are reported in the literature, but typically lies in the range of $50-200 \mathrm{~nm}$, Wang et al. 85], Nia et al. 64. Gas is stored in the network of pores and a fraction of the gas is adsorbed into the kerogen material which is the solid organic material, and a fraction of the gas is trapped inside the fractures which are the cracks or faults in the rock formation.

Shales also have low porosity, typically in the range $4-15 \%$, Vesters et al. [80], Darishchev et al. 28, and extremely low permeability which makes the movement of gas molecules very difficult, Aguilera [1, 2]. The permeability of shale rocks can vary typically between $10-2000$ nD, Darishchev et al. 28]. The pressure in shale rock formations typically varies in the range $25-60 \mathrm{MPa}$; and the temperature varies from $325-450 \mathrm{~K}$, Wang et al. 83. and Wang and Shahvali 84 .

It is not surprising therefore that conventional transport methods based upon the linear Darcy law in a continuum fails to describe the transport system adequately. In the first place, several flow regimes have to be accounted for (slip flow, tranitional flow, surface diffusion, and Knudsen flow) as well as the adsorption and desorption of the gas from the rock material. The correlation between the measured permeability and the apparent permeability that appears in such models is also important and must be modeled, Song et al. 75].

Furthermore, the system is highly pressure dependent. As the gas pressure depletes, or as the temperature changes inside the reservoirs, the pore size itself my change with pressure, and the rate of adsorption and desorption may also change. Some pores may open up, while others may close down, implying that the pore network itself may alter and therefore the permeability and porosity are not constant and must be modeled as a function of the pressure, Clarkson 
et al. 24]. Correlations for these and other physical processes in the systen must be obtained or estimated, as we will see in Sections 3 and 4 .

A realistic transport model is an important tool in the petroleum industry because it allows the future pressure distributions inside reservoirs to be estimated which assists in policy and planning, and for drilling strategies, and for estimating future recovery levels, Satter et al. [73, Hanea et al. 39, Møyner et al. 60]. In order to accurately model flow of gas in shale rock formations, both the network of induced fractures and also the porous matrix between the fractures must be considered. Unfortunately, at the current time, the modelling and simulation methods are still in their infancy, and the challenges are formidable, Aziz and Settari [7], Peaceman [66], Chen [16], Marcondes and Sepehrnoori [57, Marcondes et al. [56], Aybar et al. [6], Fernandes et al. [32], not least because there are a large number of pressure dependent modelling parameters in the system and the transport equation may be highly nonlinear because the apparent velocity $U_{a}$ and apparent diffusivity $D_{a}$ are nonlinear functions of the pressure $p(\mathbf{x}, t)$, and of the pressure gradient $\nabla p(\mathbf{x}, t)$.

The aim here is to address the first part of the general transport problem of shale gas flow described above, that is we consider single phase gas transport through unfractured rocks. We address the problem of gas transport through fractured rocks Akkutlu [3] in future studies. We concentrate on the development of a realistic gas transport model between such fractures. Some progress has been made recently in developing such transport models, Cui et al. 25] and Civan et al. 222; each model incorporates some of the physical processes, but falls short of the level of realism that is needed for current needs. Our aim is to develop a realistic transport model for shale gas flow which incorporates all the important physical transport processes in the system, and then to demonstrate its effectiveness by applying it to determining the rock properties, such as the permeability and the porosity of shale rock core samples, which is itself a very important part of research in the petroleum and oil industry, and in the 
geophysical sciences.

The rest of the paper is organised as follows. In Section 2, we summaries the important transport processes and model parameters in tight porous systems. In Section 3, we derive a new transport model for gas flow in tight porous media, for three-dimensional and for one-dimensional domains. In Section 4. we derive expressions for the various compressibility coefficients associated with all the model parameters. In Section 5, we describe the numerical procedure. In Section 6, simulation results are presented using our new transport model in order to determine rock properties through an inverse problem of matching simulations for experimental data (also known as history matching, see Oliver and Chen [65]). We discuss the results and draw conclusions in Section 7 .

\section{Flow in porous media}

\subsection{Modelling transport in porous media}

Fluid flow in porous media is a highly complex phenomenon involving many variables and many different physical processes, Bird [11], Bear [8], Mahdi et al. [53, Zhang et al. [89], Su and Davidson [76], Muljadi et al. 61], Ramakrishnan and Goode [69. Unlike conventional fluid flow, such as flow through pipes, or homogeneous turbulence, where the balance equations (Navier-Stokes) are known and only the properties of the fluid (viscosity and density) and the size of the domain and boundary conditions govern the system, in porous media the balance equations are unknown and the properties of the porous media itself, such as the porosity and the permeability, also play a leading role, Cui et al. [25], Chen et al. 15], Civan [20, Geng et al. [36], Wang and Shahvali 84]. Sometimes, you also have to deal with turbulence and with multiphase flow. It is also possible that the rock properties themselves change in response to changes in the prevailing conditions such as the pressure and the temperature; for example, the porosity may change because some pore passages may become 
blocked over time, while other passages may opened up. A general theory for flow in porous media is unknown, so we have to resort to empirical relationships, like Darcy's law, in order to model the transport through the porous media, Vafai [79, Müller-Huber et al. 62, Faybishenko et al. [31, Benzerga [9].

Mathematical models that describe the transport of gas through tight reservoirs are based upon the consideration of the amount of gas that is transported through the reservoir and the amount of gas that is retained in it. Such models appear in the form of partial differential equations (PDE's). The principal parameters upon which most models are based are the intrinsic rock permeability $(K)$ and the rock porosity $(\phi)$. An accurate determination of these properties is therefore essential for developing transport models for flow through porous media, Freeman et al. [35, Sun et al. [77, Guo et al. [38.

Darcy 27] proposed an empirical linear equation relating the convective flux (or discharge rate), $\mathbf{u}$, of the fluid, to the pressure gradient and the rock properties,

$$
\mathbf{u}=-\frac{K}{\mu} \nabla p
$$

The actual fluid velocity $\mathbf{v}$ is related to the flux through the porosity, $\phi$, by $\mathbf{v}=\mathbf{u} / \phi . p$ is the pressure, $K$ is the rock permeability, $\mu$ the viscosity.

Darcy's law yields good results for laminar flow in high porosity porous media, but in the case of high velocity flow rate it does not produce satisfactory results, Prada and Civan [68, Xu et al. 86, Guo et al. 37. Several studies have shown that the use of the mathematical models based on Darcy's law are inadequate to study transport processes through unconventional porous rocks because different non-laminar flow regimes occur in tight porous media other than the continuous (viscous) flow, Thauvin and Mohanty [78, Cui et al. 25], Civan et al. 22., Macini et al. [52]. For high velocities in porous media, inertial effects can also become significant. Sometimes a nonlinear inertial term is added in Darcy's equation, known as the Forchheimer term. This term accounts for the non- 


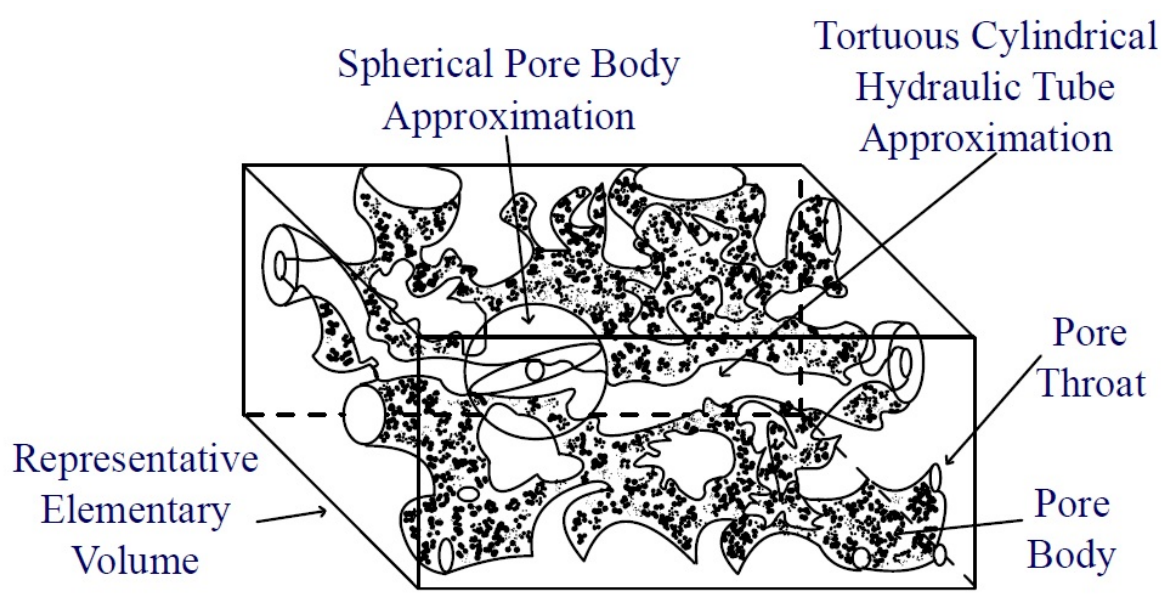

Figure 1: Representative elementary volume of a porous media which represents the structure of the solid matrix. Here the pores are given spherical geometry and flow channels are represented as cylindrical tubes. Civan 18

linear behavior of the pressure gradient. Forchheimer [34] introduced it as a quadratic term,

$$
\nabla p=-\frac{\mu}{\kappa} \mathbf{u}-\rho \mathbf{B} \cdot \mathbf{u}|\mathbf{u}|
$$

Here, $\rho$ denotes the gas density and $\mathbf{B}$ is a constant tensor of rank two (in the most general three-dimensional case). The second term on the right accounts for the non-Darcy effects due to high velocities. Many attempts have been made to modify Darcy's law through generalized models for Forchheimer's correction term, Li et al. 47, Huang et al. 42.

\subsection{Classifications of flow regimes based on Knudsen number}

Different flow regimes can be classified through the Knudsen number, Ziarani and Aguilera 91, which is defined as the ratio of the molecular mean free path $\lambda$ to the hydraulic radius $R_{h}$, of the flow channels.

$$
K_{n}=\frac{\lambda}{R_{h}}
$$


The Mean Free Path $(\lambda)$ is the average distance traveled by a gas molecule between collisions with other molecules. There exists several models for the mean free path, such as given by Loeb [49. Bird [1] derived a model, equation (2.4), which is based on variable flow head model and showed that it has advantages over the previous studies. Christou and Kokou Dadzie [17] have recently used this model in their study of direct simulation Monte Carlo methods in porous media with varying Knudsen number. The equation for the mean free path is given by,

$$
\lambda=\frac{\mu}{p} \sqrt{\frac{\pi R_{g} T}{2 M_{g}}}
$$

where $\rho$ is gas density $\left(\mathrm{kg} / \mathrm{m}^{3}\right), \mu$ is gas viscosity $(\mathrm{Pa}-\mathrm{s}), T$ is the absolute temperature $(\mathrm{K}), R_{g}=8134(\mathrm{~J} / \mathrm{kmol} / \mathrm{K})$ is the universal gas constant. $p$ is the absolute gas pressure $(\mathrm{Pa})$.

The hydraulic radius $R_{h}$ is the mean radius of a system of pores and is given by, Carman and Carman [14] and Civan [19],

$$
R_{h}=2 \sqrt{2 \tau_{h}} \sqrt{\frac{K}{\phi}},
$$

where $\tau_{h}$ is the tortuosity which is the ratio of apparent length of the effective mean hydraulic tube to the physical length of the bulk porous media, and $\phi$ is the porosity which is the fraction of volume of void spaces to the bulk volume of the porous media, see Figure 1 .

Ziarani and Aguilera [91, Rathakrishnan 70] and other researchers have followed the classification of four flow regimes based on the Knudsen number. Equations (2.3), 2.4 and 2.5 yield an expression for the Knudsen number,

$$
K_{n}=\frac{\mu}{4 p} \sqrt{\frac{\pi R_{g} T \phi}{M_{g} \tau_{h} K}} .
$$

The flow regimes are then classified as follows. Continuum (viscous) flow, Cus- 


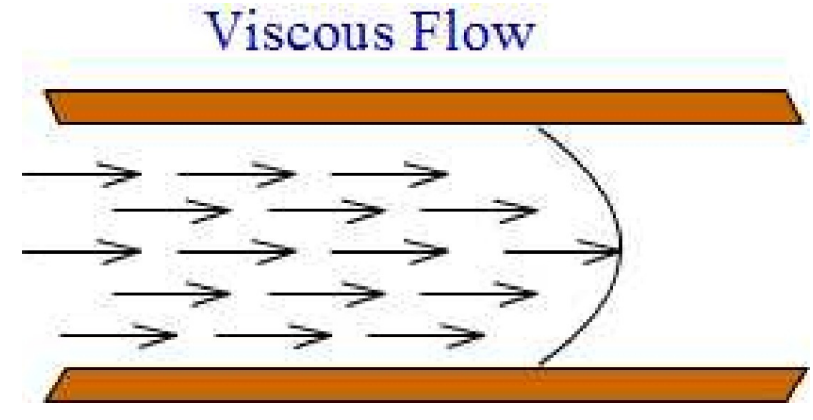

Figure 2: Viscous flow occurs when the radius of the flow channels is very large compared to the mean free path of the gas molecules. Darcy's law is used to describe the continuous (laminar) flow.

\section{Slip Flow}

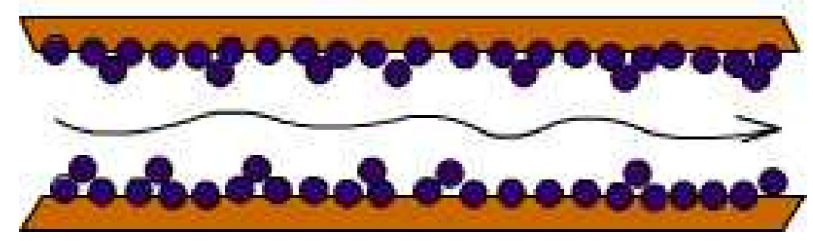

Figure 3: Slip flow occurs due to accumulation of gas molecules along pore surface. Transition flow occurs When more gas molecules collide with the pore surface movement in the gas molecules occur because of hopping. Darcy's law starts to fail in the slip and transition flow regimes.

sler [26], exists in the range where $K_{n}<0.01$, and the conventional Darcy's law can be used to describe the flow. Darcy's law was derived on the assumption of laminar flow for small Reynolds number $R e \approx O(1)$, Figure 2

Slip flow, Moghaddam and Jamiolahmady [59], exists in the range where $0.01<$ $K_{n}<0.1$. Gas molecules accumulate along the inside surface of the pore, Figure 3. and they push gas molecules towards the pore interfaces. Darcy's law, can be employed with some modifications.

Transition flow, Ziarani and Aguilera [91], exists in the range where $0.1<K_{n}<$ 10. During the slipping phenomenon, when the gas molecules collide with the gas molecules already stuck to the surface of the porous rocks, they exert some force on the molecules and some of the gas molecules leave the pore surface and 


\section{Knudsen Flow}

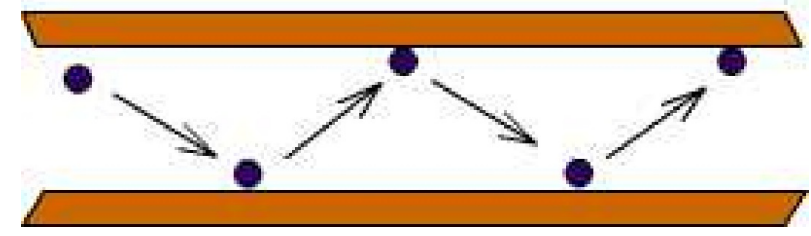

Figure 4: Knudsen diffusion or free molecular flow occurs when the radius of the flow channels is very small compared to the mean free path of the gas molecules. Darcy's law completely fails in this regime.

become a part of the continuous flow. Conventional equations fail and we must use Knudsen diffusion equations.

Knudsen (free molecular) flow, Ziarani and Aguilera 91, exists in the range where $K_{n}>10$. The mean free path of the gas molecules is much greater than the radius of the flow channels and gas molecules collide more frequently with the pore walls compared to the collision rate between gas molecules. It occurs in systems with low pressures or very tight pore throats as in the case of shale gas or coal bed methane formations, Figure 4.

\subsection{Intrinsic permeability and apparent permeability}

Gas slippage in a porous medium leads to higher than expected measured gas permeability, the apparent permeability $\mathbf{K}_{a}$, compared to the intrinsic permeability $\mathbf{K}$, Chen et al. [15]. Many correlations between intrinsic and apparent permeabilities have been proposed in the literature, Klinkenberg et al. [46], Jones et al. 45. A formula, derived from Hagen-Poiseuille-type equation, is given by Beskok and Karniadakis [10],

$$
\mathbf{K}_{a}=\mathbf{K} f\left(K_{n}\right)
$$


where $f\left(K_{n}\right)$ is the flow condition function and is given by

$$
f\left(K_{n}\right)=\left(1+\sigma K_{n}\right)\left(1+\frac{4 K_{n}}{1-b K_{n}}\right)
$$

where $\sigma$ is called the rarefaction coefficient correlation. Different correlations for $\sigma$ have been proposed by Beskok and Karniadakis [10, Civan et al. 22, Freeman et al. [35. In this work we use the correlation proposed by Civan [19,

$$
\sigma=\sigma_{o}\left(\frac{K_{n}^{b_{\sigma}}}{K_{n}^{b_{\sigma}}+a_{\sigma}}\right)=\sigma_{o}\left(1+\frac{a_{\sigma}}{K_{n}^{b_{\sigma}}}\right)^{-1}
$$

where $a_{\sigma}$ and $b_{\sigma}$ are empirical constants, and $b$ in equation $(2.8)$ is called the slip factor.

\subsection{Gas adsorption isotherm}

As the gas is transported through the tight pore network, some of the gas adheres (clings) to pore surfaces due to the diffusion of gas molecules. Cui et al. 25] and Civan et al. 22 developed a formula for estimating the amount of adsorbed gas based on Langmuir isotherms, which will be discussed further in section 4.2.

\section{A transient transport model for gas flow in tight porous media}

The approach in developing a realistic transport model is to include as many physical processes in the system as possible. Furthermore, a crucial part of the new model is to allow all of the model parameters to be functions of the pressure. For completeness, we first develop a general three-dimensional model, and then to obtain a one-dimensional model from it which will be used in subsequent application. The new model encompasses all the different flow regimes through the Knudsen number. The turbulent effects at high velocities are included in the model by a Forchheimer's correction term. 


\subsection{Conservation of mass and momentum}

Mass conservation of gas transport through the tight porous media is described by including the loss of mass of gas by adsorption per unit bulk volume of porous media and per unit time (the second term on the left below) and is given by,

$$
\frac{\partial(\rho \phi)}{\partial t}+\frac{\partial[(1-\phi) q]}{\partial t}=-\nabla \cdot(\rho \mathbf{u})+Q
$$

$q$ is the mass of gas absorbed per solid volume of rock. $Q$ is some external source.

Momentum conservation of gas flowing through porous media is described by a modified Darcy's law and is given by Evans and Civan 30.

$$
\begin{aligned}
-(\nabla p-\rho g \nabla H) & =\mu \mathbf{K}_{a}^{-1} \cdot \mathbf{u}+\rho \mathbf{B}|\mathbf{u}| \cdot \mathbf{u} \\
& =\mu \mathbf{K}_{a}^{-1}\left(\mathbf{I}+\frac{\rho}{\mu} \mathbf{B} \mathbf{K}_{a}|\mathbf{u}|\right) \cdot \mathbf{u}
\end{aligned}
$$

where $\rho\left(\mathrm{kg} / \mathrm{m}^{3}\right)$ is the density, $\mathbf{u}\left(\mathrm{m}^{3} / \mathrm{s} / \mathrm{m}^{2}\right)$ is the volumetric flux, $\mu$ (Pa s) is the dynamic viscosity of the flowing gas, $g\left(\mathrm{~m}^{2} / \mathrm{s}\right)$ is the magnitude of the gravitational acceleration vector, $H(\mathrm{~m})$ is the depth function, $\mathbf{K}_{a}\left(\mathrm{~m}^{2}\right)$ denotes the apparent permeability tensor of the rock, $p$ is the pressure, and $\mathbf{B}$ represents the inertial and turbulence effects where the velocity is high, see equation 2.2 .

Setting $\mathbf{F}^{-1}=\left(\mathbf{I}+\frac{\rho}{\mu} \mathbf{K}_{a} \mathbf{B}|\mathbf{u}|\right)$, where $\mathbf{I}$ is the identity matrix, we obtain,

$$
\mathbf{F}=\left(\mathbf{I}+\frac{\rho}{\mu} \mathbf{K}_{a} \mathbf{B}|\mathbf{u}|\right)^{-1}
$$

then equation $(3.2)$ can be written as

$$
-(\nabla p-\rho g \nabla H)=\mu\left(\mathbf{F K}_{a}\right)^{-1} \cdot \mathbf{u}
$$


from which it follows that,

$$
\mathbf{u}=-\frac{1}{\mu}\left(\mathbf{F K}_{a}\right) \cdot(\nabla p-\rho g \nabla H) .
$$

Combining equations (3.1) and 3.5, and after rearranging, we obtain

$$
\begin{aligned}
\frac{\partial(\rho \phi)}{\partial t}+\frac{\partial[(1-\phi) q]}{\partial t} & =\frac{\rho}{\mu}\left(\mathbf{F K}_{a}\right): \nabla \nabla p+\nabla \cdot\left(\frac{\rho}{\mu}\left(\mathbf{F K}_{a}\right)\right) \cdot \nabla p \\
& -\nabla \cdot\left(\frac{\rho^{2} g}{\mu}\left(\mathbf{F} \mathbf{K}_{a}\right) \cdot \nabla H\right)+Q
\end{aligned}
$$

\subsection{The pressure equation}

When pressure is applied, it changes the physical properties of the system. The changes in the physical quantities is measured in terms of compressibility coefficients. The compressibility of some parameter, $\gamma(p)$, is the relative change in $\gamma(p)$ in response to the change in the pressure. The isothermal coefficient of compressibility, $\zeta_{\gamma}(p)$, of property $\gamma$ is defined as,

$$
\zeta_{\gamma}(p)=\frac{1}{\gamma} \frac{\partial \gamma}{\partial p}=\frac{\partial}{\partial p}(\ln \gamma)
$$

Thus the isothermal coefficient of compressibility for fluid density $\rho$ is,

$$
\zeta_{\rho}(p)=\frac{1}{\rho} \frac{\partial \rho}{\partial p}=\frac{1}{p}-\frac{1}{Z} \frac{\partial Z}{\partial p}=\frac{1}{p}-\zeta_{Z}(p) ; \quad \text { where } \quad \rho=\frac{p M_{g}}{Z R_{g} T} .
$$

For quantities, such as the fluid viscosity $(\mu)$, and the rock porosity $(\phi)$, we assume an exponential integral relation. Thus

$$
\zeta_{\mu}(p)=\frac{1}{\mu} \frac{\partial \mu}{\partial p} ; \quad \text { where } \mu=\mu_{0} \exp \left(\int_{p_{0}}^{p} \zeta_{\mu}(p) d p\right)
$$

and,

$$
\zeta_{\phi}(p)=\frac{1}{\phi} \frac{\partial \phi}{\partial p} ; \quad \text { where } \phi=\phi_{0} \exp \left(\int_{p_{0}}^{p} \zeta_{\phi}(p) d p\right)
$$


For a matrix (tensor) quantity like the rock permeability, $\mathbf{K}_{a}$, we define $\zeta_{\mathbf{K}_{a}}$ as follows,

$$
\zeta_{\mathbf{K}_{a}}(p) \mathbf{I}=\mathbf{K}_{a}^{-1} \frac{\partial}{\partial p}\left(\mathbf{K}_{a}\right) ; \quad \text { where } \mathbf{K}_{a}=\left(\mathbf{K}_{a}\right)_{0} \exp \left(\int_{p_{0}}^{p} \zeta_{\mathbf{K}_{a}}(p) d p\right)
$$

Other compressibility coefficients are derived as follows,

$$
\begin{gathered}
\zeta_{1}(p)=\frac{1}{\rho \phi} \frac{\partial(\rho \phi)}{\partial p}=\frac{1}{\rho} \frac{\partial \rho}{\partial p}+\frac{1}{\phi} \frac{\partial \phi}{\partial p}=\zeta_{\rho}(p)+\zeta_{\phi}(p) \\
\zeta_{2}(p)=\frac{1}{(1-\phi) q} \frac{\partial[(1-\phi) q}{\partial p}=\zeta_{q}(p)-\left(\frac{\phi}{1-\phi}\right) \zeta_{\phi}(p) \\
\zeta_{3}(p) \mathbf{I}=\left(\frac{\rho}{\mu}\left(\mathbf{F} \mathbf{K}_{a}\right)\right)^{-1} \frac{\partial}{\partial p}\left(\frac{\rho}{\mu}\left(\mathbf{F} \mathbf{K}_{a}\right)\right) \\
=\left[\zeta_{\rho}(p)-\zeta_{\mu}(p)+\zeta_{\left(\mathbf{F} \mathbf{K}_{a}\right)}(p)\right] \mathbf{I} \\
\Rightarrow \zeta_{3}(p)=\zeta_{\rho}(p)-\zeta_{\mu}(p)+\zeta_{(\mathbf{F K}}(p),
\end{gathered}
$$

where,

$$
\begin{aligned}
\zeta_{\left(\mathbf{F K}_{a}\right)}(p) \mathbf{I} & =\left(\mathbf{F} \mathbf{K}_{a}\right)^{-1} \frac{\partial}{\partial p}\left(\mathbf{F} \mathbf{K}_{a}\right) \\
& =\left[\mathbf{K}_{a}^{-1} \zeta_{\mathbf{F}}(p) \mathbf{K}_{a}+\zeta_{\mathbf{K}_{a}}(p)\right] \mathbf{I} \\
\Rightarrow \zeta_{\left(\mathbf{F K}_{a}\right)}(p) & =\zeta_{\mathbf{F}}(p)+\zeta_{\mathbf{K}_{a}}(p) .
\end{aligned}
$$

Equation 3.14 then becomes

$$
\zeta_{3}(p)=\zeta_{\rho}(p)-\zeta_{\mu}(p)+\zeta_{\mathbf{F}}(p)+\zeta_{\mathbf{K}_{a}}(p) .
$$


From equation (3.3), we have

$$
\begin{aligned}
\mathbf{F}^{-1}-\mathbf{I} & =\frac{\rho}{\mu} \mathbf{K}_{a} \mathbf{B}|\mathbf{u}| \\
\zeta_{\mathbf{F}}(p) \mathbf{I} & =\mathbf{F}^{-1} \frac{\partial}{\partial p} \mathbf{F}
\end{aligned}
$$

Using the general relation, $\frac{\partial}{\partial p} \mathbf{A}^{-1}=-\mathbf{A}^{-1} \frac{\partial \mathbf{A}}{\partial p} \mathbf{A}^{-1}$, for some tensor $\mathbf{A}$, this leads to,

$$
\begin{aligned}
\zeta_{\mathbf{F}}(p) \mathbf{I} & =-\mathbf{F}^{-1} \mathbf{F}\left(\frac{\partial}{\partial p} \mathbf{F}^{-1}\right) \mathbf{F} \\
& =-\frac{\rho}{\mu}\left(\mathbf{K}_{a} \mathbf{B}\right)|\mathbf{u}|\left[\left(\frac{\rho}{\mu}\right)^{-1} \frac{\partial}{\partial p}\left(\frac{\rho}{\mu}\right) \mathbf{I}+\left(\mathbf{K}_{a} \mathbf{B}\right)^{-1} \frac{\partial}{\partial p}\left(\mathbf{K}_{a} \mathbf{B}\right)+\frac{1}{|\mathbf{u}|} \frac{\partial|\mathbf{u}|}{\partial p} \mathbf{I}\right] \mathbf{F}
\end{aligned}
$$

Substituting equation (3.17) in to equation 3.19), we obtain

$$
\zeta_{\mathbf{F}}(p) \mathbf{I}=(\mathbf{F}-\mathbf{I})\left[\zeta_{\rho}(p)-\zeta_{\mu}(p)+\zeta_{\left(\mathbf{K}_{a} \mathbf{B}\right)}(p)+\zeta_{|\mathbf{u}|}(p)\right] .
$$

The compressibility coefficient $\zeta_{\left(\mathbf{K}_{a} \mathbf{B}\right)}(p)$ can be expressed as the sum of $\zeta_{\mathbf{K}_{a}}$ and $\zeta_{\mathrm{B}}$ as follows,

$$
\begin{aligned}
\zeta_{\left(\mathbf{K}_{a} \mathbf{B}\right)}(p) \mathbf{I} & =\left(\mathbf{K}_{a} \mathbf{B}\right)^{-1} \frac{\partial}{\partial p}\left(\mathbf{K}_{a} \mathbf{B}\right) \\
& =\left[\mathbf{B}^{-1} \zeta_{\mathbf{K}_{a}}(p) \mathbf{B}+\zeta_{\mathbf{B}}(p)\right] \mathbf{I} \\
\Rightarrow \zeta_{\left(\mathbf{K}_{a} \mathbf{B}\right)}(p) & =\zeta_{\mathbf{K}_{a}}(p)+\zeta_{\mathbf{B}}(p)
\end{aligned}
$$

where,

$$
\zeta_{\mathbf{B}}(p) \mathbf{I}=\mathbf{B}^{-1} \frac{\partial \mathbf{B}}{\partial p}
$$


Substituting equation (3.21) into equation (3.20), we obtain

$$
\zeta_{\mathbf{F}}(p) \mathbf{I}=(\mathbf{F}-\mathbf{I})\left[\zeta_{\rho}(p)-\zeta_{\mu}(p)+\zeta_{\mathbf{K}_{a}}(p)+\zeta_{\mathbf{B}}(p)+\zeta_{|\mathbf{u}|}(p)\right]
$$

Substituting equation (3.23) into equation 3.15 and then into equation (3.14), we obtain

$$
\begin{aligned}
\zeta_{3}(p) \mathbf{I} & =\left(\zeta_{\rho}(p)+\zeta_{\mathbf{K}_{a}}(p)-\zeta_{\mu}(p)\right) \mathbf{I}+\zeta_{\mathbf{F}}(p) \mathbf{I} \\
& =\mathbf{F}\left[\zeta_{\rho}(p)+\zeta_{\mathbf{K}_{a}}(p)-\zeta_{\mu}(p)\right]+(\mathbf{F}-\mathbf{I})\left(\zeta_{\mathbf{B}}(p)+\zeta_{|\mathbf{u}|}(p)\right)
\end{aligned}
$$

Using equation (2.7), we have,

$$
\begin{aligned}
\zeta_{\mathbf{K}_{a}}(p) \mathbf{I} & =\mathbf{K}_{a}^{-1} \frac{\partial}{\partial p} \mathbf{K}_{a}=(f \mathbf{K})^{-1} \frac{\partial}{\partial p} f \mathbf{K} \\
& =\left[\zeta_{f}(p)+\zeta_{\mathbf{K}}(p)\right] \mathbf{I} \\
\Rightarrow \zeta_{\mathbf{K}_{a}}(p) & =\zeta_{f}(p)+\zeta_{\mathbf{K}}(p)
\end{aligned}
$$

where,

$$
\zeta_{\mathbf{K}}(p) \mathbf{I}=\mathbf{K}^{-1} \frac{\partial}{\partial p} \mathbf{K} ; \quad \text { and } \mathbf{K}=(\mathbf{K})_{0} \exp \left(\int_{p_{0}}^{p} \zeta_{\mathbf{K}}(p) d p\right)
$$

and,

$$
\zeta_{f}(p) \mathbf{I}=\left(f\left(\mathbf{K}_{n}\right)\right)^{-1} \frac{\partial}{\partial p} f\left(\mathbf{K}_{n}\right)
$$

and,

$$
\begin{aligned}
\zeta_{|\mathbf{u}|}(p) & =|\mathbf{u}|^{-1} \frac{\partial}{\partial p}|\mathbf{u}| \\
& =\frac{1}{\mathbf{u} \cdot \mathbf{u}}\left(\mathbf{u} \cdot \frac{\partial \mathbf{u}}{\partial p}\right) .
\end{aligned}
$$

Note that all compressibility coefficients are combinations of four basic ones, namely, $\zeta_{\rho}, \zeta_{K}, \zeta_{f}$, and $\zeta_{\mu}$. 
From equations $(3.12)$ and $(3.13)$, we derive the following expressions:

$$
\frac{\partial(\rho \phi)}{\partial t}=\frac{\partial(\rho \phi)}{\partial p} \frac{\partial p}{\partial t}=\rho \phi \zeta_{1}(p) \frac{\partial p}{\partial t}
$$

and

$$
\frac{\partial(1-\phi) q}{\partial t}=\frac{\partial(1-\phi) q}{\partial p} \frac{\partial t}{\partial t}=(1-\phi) q \zeta_{2}(p) \frac{\partial p}{\partial t} .
$$

Furthermore, we note that,

$$
\begin{aligned}
\nabla \cdot\left(\frac{\rho}{\mu}\left(\mathbf{F} \mathbf{K}_{a}\right)\right) & \equiv\left(\nabla p \frac{\partial}{\partial p}\right) \cdot\left(\frac{\rho}{\mu}\left(\mathbf{F} \mathbf{K}_{a}\right)\right) \\
& =\zeta_{3}(p) \nabla p \cdot\left(\frac{\rho}{\mu}\left(\mathbf{F} \mathbf{K}_{a}\right)\right)
\end{aligned}
$$

Substituting equations (3.29), 3.30) and (3.31) into (3.6), we obtain

$$
\begin{aligned}
\rho \phi \zeta_{1}(p) \frac{\partial p}{\partial t} & +(1-\phi) q \zeta_{2}(p) \frac{\partial p}{\partial t}= \\
& \frac{\rho}{\mu}\left(\mathbf{F} \mathbf{K}_{a}\right): \nabla \nabla p+\zeta_{3}(p) \nabla p \cdot\left(\frac{\rho}{\mu}\left(\mathbf{F} \mathbf{K}_{a}\right)\right) \cdot \nabla p \\
& -\nabla \cdot\left(\frac{\rho^{2} g}{\mu}\left(\mathbf{F K}_{a}\right) \cdot \nabla H\right)+Q
\end{aligned}
$$

We define the apparent diffusivity $\mathbf{D}_{a}\left(\mathrm{~m}^{2} / \mathrm{s}\right)$ as,

$$
\mathbf{D}_{a}(p)=\frac{\rho \mathbf{F} \mathbf{K}_{a}}{\mu} \chi
$$

where

$$
\chi^{-1}=\left[\phi \zeta_{1}(p)+(1-\phi) \frac{q}{\rho} \zeta_{2}(p)\right] .
$$


Using equations (3.31), 3.33) and (3.34) in equation 3.32 we obtain,

$$
\begin{aligned}
\frac{\partial p}{\partial t} & =\mathbf{D}_{a}(p): \nabla \nabla p+\zeta_{3}(p) \nabla p \cdot \mathbf{D}_{a}(p) \cdot \nabla p \\
& -\rho g \mathbf{D}_{a}: \nabla \nabla H-\rho g \zeta_{3}(p) \nabla p \cdot \mathbf{D}_{a} \cdot \nabla H-\nabla(\rho g) \cdot \mathbf{D}_{a} \cdot \nabla H+\chi Q
\end{aligned}
$$

Equation (3.35) is the most general transport equation for gas flow in threedimensional porous media which can be derived under the present assumptions, which includes gravity and a general source term.

The transport model (3.35) has a number of important features. It incorporates the various flow regimes that occur in the porous media, and the high velocity effects are included through the Forchheimer's nonlinear correction term. The turbulence factor $\mathbf{B}$ is considered as a function of $\mathbf{K}_{a}, \phi$, and $\tau$. Moreover, the parameters $\phi, \mu, \rho$ are functions of pressure $p$. Hence the model 3.35 has nonlinear coefficients $D_{a}$ and $U_{a}$.

\subsection{No gravity}

Under the assumption of no gravity $(g=0)$, equation 3.35 becomes

$$
\frac{\partial p}{\partial t}=\mathbf{D}_{a}(p): \nabla \nabla p+\nabla p \cdot \mathbf{D}_{a}(p) \zeta_{3}(p) \cdot \nabla p+\psi Q .
$$

\subsection{No source term}

Under the further assumption of no source/sink term $(Q=0)$, equation 3.36 becomes

$$
\frac{\partial p}{\partial t}=\mathbf{D}_{a}(p): \nabla \nabla p+\nabla p \cdot \mathbf{D}_{a}(p) \zeta_{3}(p) \cdot \nabla p .
$$




\subsection{One-dimensional equation}

Under the further assumption of one-dimensional flow, equation (3.37) becomes

$$
\frac{\partial p}{\partial t}+U_{a}\left(p, p_{x}\right) \frac{\partial p}{\partial x}=D_{a}(p) \frac{\partial^{2} p}{\partial x^{2}}
$$

where, $U_{a}(\mathrm{~m} / \mathrm{s})$ is called the apparent convective flux (or convective velocity) and is defined by,

$$
U_{a}=-\zeta_{3}(p) D_{a}(p) \frac{\partial p}{\partial x}
$$

and

$$
D_{a}(p)=\frac{\rho}{\mu} \frac{F K_{a}}{\left(\rho \phi \zeta_{1}(p)+(1-\phi) q \zeta_{2}(p)\right)},
$$

is called the apparent diffusivity, where $F$ and $K_{a}$ are now scalar quantities.

Similar models were considered by Malkovsky et al. [55, Liang et al. [48, Civan et al. 22]. However, these models do not include the high velocity corrections, and some of the models make other approximations such as constant model parameters.

\subsection{Non-dimensional steady state pressure equation}

A steady state model can be obtained by setting $\frac{\partial p}{\partial t}=0$ in equation 3.38 to yield

$$
U_{a}\left(p, p_{x}\right) \frac{\partial p}{\partial x}=D_{a}(p) \frac{\partial^{2} p}{\partial x^{2}},
$$

which can be rearranged to yield,

$$
L_{a}\left(p, p_{x}\right) \frac{\partial p}{\partial x}=\frac{\partial^{2} p}{\partial x^{2}}
$$

where,

$$
L_{a}=-\zeta_{3}(p) \frac{\partial p}{\partial x}
$$


where $\zeta_{3}(p)$ is given by equation 3.16 .

For the case of transport through a core sample of size $x_{D}$, with pressure given at the inlet and outlet boundaries, a dimensionless form of steady state equation can be obtained by considering the following dimensionless variables,

$$
x_{D}=\frac{x}{L} \quad \text { and } \quad p_{D}\left(x_{D}\right)=\frac{p(x)-p_{d}}{p_{u}-p_{d}}
$$

which yields,

$$
L_{a}\left(p_{D},\left(p_{D}\right)_{x}\right) \frac{\partial p_{D}}{\partial x_{D}}=\frac{\partial^{2} p_{D}}{\partial x_{D}^{2}}, \quad 0 \leq x_{D} \leq 1
$$

Furthermore, a non-dimensional transient pressure equation can be obtained by introducing the dimensionless variables,

$$
\begin{gathered}
x_{D}=\frac{x}{L}, \quad t_{D}=\frac{t}{t_{0}}, \quad p_{D}\left(x_{D}, t_{D}\right)=\frac{p(x, t)-p_{d}(t)}{p_{u}(0)-p_{d}(0)} . \\
t_{0}=\frac{L^{2}}{D_{0}}, \quad U_{D}=\frac{U_{a}}{U_{0}}, \quad D_{D}=\frac{D_{a}}{D_{0}}, \quad P e=\frac{L U_{0}}{D_{0}} .
\end{gathered}
$$

The transient equation 3.38 then reduces to the following dimensionless form,

$$
\frac{\partial p_{D}}{\partial t_{D}}+P e U_{D} \frac{\partial p_{D}}{\partial x_{D}}=D_{D} \frac{\partial^{2} p_{D}}{\partial x_{D}^{2}}, \quad 0 \leq x_{D} \leq 1, \quad t_{D}>0
$$

where $P e$ is the Peclet number, $D_{o}$ and $U_{o}$ are values of the diffusivity coefficient $D_{a}$ and the convective flux $U_{a}$ at some specific pressure, and $p_{u}(t)$ and $p_{d}(t)$ are pressures in the upstream and the downstream reservoirs. 


\section{Compressibility coefficients}

\subsection{Parameters in the new transport model}

In application, we will be dealing with one-dimensional flow, with zero gravity, and with no external source, equation (3.38. $U_{a}, D_{a}, \chi, F, \zeta_{1}(p), \zeta_{2}(p)$, and $\zeta_{3}$ are defined in equations (3.39), (3.40), 3.34), (3.3), 3.12), (3.13), and (3.24), respectively. For transport through a reservoir, it requires only boundary and initial conditions to solve for the pressure distribution $p(x, t)$.

A complication is the appearance of so many compressibility coefficients each of which must be known or modelled in order for this system to be solvable. We list these models below.

The real gas deviation factor $(Z)$ is calculated from Mahmoud [54,

$$
\begin{aligned}
Z & =a_{Z} p_{r}^{2}+b_{Z} p_{r}+c_{Z}, \\
a_{Z} & =0.702 \exp \left(-2.5 t_{r}\right), \\
b_{Z} & =-5.524 \exp \left(-2.5 t_{r}\right), \\
c_{Z} & =0.044 t_{r}^{2}-0.164 t_{r}+1.15,
\end{aligned}
$$

where $p_{r}=p / p_{c}$ is the reduced pressure and $t_{r}=T / T_{c}$, is the reduced temperature, and $p_{c}$ and is critical pressure, and $t_{c}$, is the critical temperature - these quantities are assumed known. The density, $\rho$, of real gases is defined by the relationship, $\rho=\frac{p M_{g}}{Z R_{g} t}$, which can be re-expressed as,

$$
\rho=\frac{p_{r} M_{g}}{t_{r} R_{g} Z\left(p_{r}\right)}
$$

The compressibility coefficient of the gas density, $\zeta_{\rho}$, is thus given by,

$$
\zeta_{\rho}(p)=\frac{d(\ln \rho)}{d p}=\frac{1}{p}-\frac{1}{p_{c} Z}\left(\frac{2 a_{Z} p}{p_{c}}+b_{Z}\right) .
$$


Setting $\zeta_{Z}(p)=\frac{1}{Z} \frac{\partial Z}{\partial p}$, this re-arranges to,

$$
\zeta_{\text {gas }}(p)=\zeta_{\rho}(p)+\zeta_{z}(p)=\frac{1}{p}
$$

The model for the gas dynamic viscosity, $\mu$, used here is, Mahmoud [54,

$$
\begin{aligned}
\mu & =\mu_{S_{c}} \exp \left(A_{\mu} \rho^{B_{\mu}}\right), \\
A_{\mu} & =3.47+1588 t^{-1}+0.0009 M_{g} \\
B_{\mu} & =1.66378-0.04679 A_{\mu} \\
\mu_{S c} & =\frac{1}{10.5^{4}}\left[\frac{M_{g}^{3} p_{c}^{4}}{t_{c}}\right]^{1 / 6}
\end{aligned}
$$

The compressibility coefficient of gas viscosity, $\zeta_{\mu}$, is then given by

$$
\zeta_{\mu}(p)=\frac{d}{d p} \ln \mu=A_{\mu} B_{\mu} \rho^{B_{\mu}} \zeta_{\rho}(p)
$$

For the porosity, $\phi$, we use the correlation, Bockstiegel [12], Walsh and Brace [81, Regnet et al. [71, Zheng et al. [90],

$$
\phi=a_{\phi} \exp \left(-b_{\phi} p^{c_{\phi}}\right)
$$

where $a_{\phi}, b_{\phi}$, and $c_{\phi}$ are empirical constants, assumed known. The compressibility coefficient of the porosity, $\zeta_{\phi}$, is given by,

$$
\zeta_{\phi}(p)=\frac{d}{d p} \ln \phi=-b_{\phi} c_{\phi} p^{c_{\phi}-1}
$$

The Intrinsic Permeability, $K$, is a fundamental property of the reservoir rocks. Different models and empirical relations have been proposed to estimate the permeability of reservoir rocks. One of the most commonly used relations is the Kozeny-Carman equation which is derived on the assumption of continuous 
flow of fluid through a bundle of parallel tubes of constant diameter, see $\mathrm{Xu}$ and $\mathrm{Yu}$ [87. This equation gives good results for the homogeneous porous media with the laminar flow, but it fails to accurately predict the permeability of heterogeneous reservoirs with a complex network of pores. It also fails for reservoirs with very low porosity and low permeability values. Here, we use a modified Power-Law form of the Kozeny-Carman equation, Civan [21] ,

$$
\sqrt{\frac{K}{\phi}}=\Gamma_{K C}\left(\frac{\phi}{\alpha_{K C}-\phi}\right)^{\beta_{K C}}
$$

where $\alpha_{K C}, \beta_{K C}$, and $\Gamma_{K C}$ are empirical constants, with $\phi<\alpha_{K C} \leq 1$, and $0 \leq \beta_{K C}<\infty$, and $\Gamma_{K C} \geq 0$. This can be rearranged to yield,

$$
K=\Gamma_{K C}^{2} \frac{\phi^{2 \beta_{K C}+1}}{\left(\alpha_{K C}-\phi\right)^{2 \beta_{K C}}} .
$$

The compressibility coefficient for the intrinsic permeability, $\zeta_{K}$, is given by,

$$
\zeta_{K}(p)=\frac{d(\ln K)}{d p}=-b_{\phi} c_{\phi} p^{c_{\phi}-1}\left(1+\frac{2 \alpha_{K C} \beta_{K C}}{\alpha_{K C}-\phi}\right) .
$$

Tortuosity, $\tau$, is a measure of the geometric complexity of the pore network and inter-connectivity, and it is defined as the ratio of the length of a typical streamline, or path, between two boundaries, to the the bulk length of the reservoir rock. There exists several relations between tortuosity and porosity but none of them works for all situations, see Matyka et al. [58. We use the following correlation for the tortuosity, Matyka et al. [58],

$$
\tau=1+a_{\tau}(1-\phi(p)),
$$

where $a_{\tau}$ is a fitting constant. Tortuosity $\tau$ is a decreasing function of $\phi$ because $d \tau / d \phi=-a_{\tau}<0$. Note that as $p \rightarrow 0$ then $\phi \rightarrow a_{\phi}$, and, therefore $\tau \rightarrow$ $1+a_{\tau}\left(1-a_{\phi}\right)$. The compressibility coefficient of tortuosity $\zeta_{\tau}$ is given by the 
expression,

$$
\zeta_{\tau}(p)=\frac{d}{d p} \ln \tau=-a_{\tau} \frac{\phi}{\tau} \zeta_{\phi}(p)
$$

The turbulence correction factor, $B$ (which is a scalar in one-dimension), is modelled by, Zhang [88, Thauvin and Mohanty [78, and Macini et al. 52,

$$
B\left(\phi, K_{a}, \tau\right)=\frac{a_{B} \tau^{b_{B}}}{K_{a}^{c_{B}} \phi^{d_{B}}}
$$

where, $a_{B}, b_{B}, c_{B}$ and $d_{B}$ are empirical constants. The Compressibility coefficient of turbulence factor $\zeta_{b}$ is given by,

$$
\zeta_{B}(p)=\frac{d}{d p} \ln B=b_{B} \zeta_{\tau}(p)-c_{B} \zeta_{K_{a}}(p)-d_{B} \zeta_{\phi}(p) .
$$

The control factor, $F$, introduced in Section 3 is given by

$$
F=\left[1+\frac{B \rho}{\mu} K_{a}|u|\right]^{-1}
$$

The compressibility coefficient for the control factor $\zeta_{F}$ is given by,

$$
\zeta_{F}(p)=(F-1)\left[\zeta_{\rho}(p)-\zeta_{\mu}(p)+\zeta_{K_{a}}(p)+\zeta_{B}(p)+\zeta_{|u|}(p)\right],
$$

where $\zeta_{K_{a}}(p)=\zeta_{K}(p)+\zeta_{f}(p)$, and $\zeta_{K}(p)$ is given by equation 4.17) and $\zeta_{f}(p)$ is defined in equation (3.27).

\subsection{Gas adsorption isotherm}

The shale adsorbs a portion of the gas, the amount retained is a dynamic process depending upon the pressure and the adsorbant, e.g. kerogen, in the shale. As the pressure in the shale decreases, the gas is desorbed in to the pore network. The relationship between pressure and the volume of the adsorbed gas 
is described by the desorption isotherm. The most commonly used relation to estimate the amount of gas released is the Langmuir Isotherm Formula, Foo and Hameed [33. Cui et al. 25], and Civan et al. 22] used the following formula, which is based upon the Langmuir adsorption isotherm,

$$
\begin{aligned}
q_{a} & =\frac{q_{L} p}{p_{L}+p}, \\
q & =\frac{\rho_{s} M_{g}}{V_{s t d}} q_{a}
\end{aligned}
$$

where $\rho_{s}\left(\mathrm{~kg} / \mathrm{m}^{3}\right)$ denotes the material density of the porous sample, $q\left(\mathrm{~kg} / \mathrm{m}^{3}\right)$ is the mass of gas adsorbed per solid volume, $q_{a}\left(\mathrm{std}^{3} / \mathrm{kg}\right)$ is the standard volume of gas adsorbed per solid mass, $q_{L}\left(\operatorname{std~} \mathrm{m}^{3} / \mathrm{kg}\right)$ is the Langmuir gas volume, $V_{\text {std }}\left(\operatorname{std~}^{3} / \mathrm{kmol}\right)$ is the molar volume of gas at standard temperature $(273.15 \mathrm{~K})$ and pressure $(101,325 \mathrm{~Pa}), p(\mathrm{~Pa})$ is the gas pressure, $p_{L}(\mathrm{~Pa})$ is the Langmuir gas pressure, and $M_{g}(\mathrm{~kg} / \mathrm{kmol})$ is the molecular weight of gas. (Half of the gas molecules occupies the empty spaces along the surface of the pores at the Langmuir pressure $p_{L}$.)

The compressibility coefficient of the adsorbed gas $\zeta_{q}$ is given by,

$$
\zeta_{q}(p)=\frac{p_{L}}{p\left(p_{L}+p\right)}
$$

$\zeta_{q}(p)$ decreases with increasing pressure. It implies that $\zeta_{q}(p)$ is limited by the number of empty spaces available at the pore surface.

\subsection{Compressibility Coefficient of Knudsen number}

The compressibility coefficient of the mean free path $\zeta_{\lambda}$ is given by

$$
\zeta_{\lambda}(p)=\zeta_{\mu}(p)-\zeta_{\rho}(p)
$$


Substituting equations 4.15 and 4.18 in to equation 2.5 , we obtain

$$
R_{h}=2 \Gamma_{K C} \sqrt{2+2 a_{\tau}(1-\phi)}\left(\frac{\phi}{\alpha_{K C}-\phi}\right)^{\beta_{K C}} .
$$

Note that $R_{h}$ increases with increase in pressure.

The compressibility coefficient of the hydraulic radius, $\zeta_{R}$, is given by,

$$
\zeta_{R}(p)=\frac{d\left(\ln R_{h}\right)}{d p}=\frac{1}{2}\left[\zeta_{\tau}(p)+\zeta_{K}-\zeta_{\phi}(p)\right]
$$

In general, $\zeta_{R}$ decreases with the increase in pressure.

A formula for the Knudsen number is obtained by substituting equations 2.4 and 4.28 into equation 2.3 ,

$$
K_{n}=\frac{1}{2 \Gamma_{K C}} \sqrt{\frac{\pi}{2 R_{g} T}} \frac{\mu}{\rho \sqrt{2+2 a_{\tau}(1-\phi)}}\left(\frac{\alpha_{K C}-\phi}{\phi}\right)^{\beta_{K C}}
$$

The compressibility coefficient of Knudsen Number $\zeta_{K_{n}}$ is given by

$$
\begin{aligned}
\zeta_{K_{n}}(p) & =\frac{d\left(\ln K_{n}\right)}{d p}=\zeta_{\lambda}(p)-\zeta_{R}(p) \\
& =\zeta_{\mu}(p)-\zeta_{\rho}(p)+\frac{1}{2}\left[\zeta_{\phi}(p)-\zeta_{\tau}(p)-\zeta_{K}(p)\right] .
\end{aligned}
$$

$\zeta_{K_{n}}$ has negative values.

\subsection{Intrinsic permeability and apparent permeability}

Using equations 2.7, 2.8, and 2.9), the compressibility coefficient for $K_{a}$, is given by

$$
\zeta_{K_{a}}=\zeta_{K}+\zeta_{f}
$$

$\zeta_{K}$ is given by equation (4.17). To compute $\zeta_{f}$, from equation 2.8 we have

$$
\zeta_{f}=\frac{d(\ln (f))}{d p}=K_{n} \frac{d(\ln (f))}{d K_{n}} \frac{1}{K_{n}} \frac{d K_{n}}{d p}=\zeta_{K_{n}} K_{n} \frac{d(\ln (f))}{d K_{n}}
$$


where,

$$
\begin{aligned}
K_{n} \frac{d(\ln (f))}{d K_{n}}= & \left(\frac{\sigma K_{n}}{1+\sigma K_{n}}\right)\left(1+\frac{K_{n}}{\sigma} \frac{d \sigma}{d K_{n}}\right) \\
& +\frac{\left(4-b_{S F}\right) K_{n}}{\left(1+\left(4-b_{S F}\right) K_{n}\right)}+\frac{b_{S F} K_{n}}{1-b_{S F} K_{n}}
\end{aligned}
$$

In the limit of continuous (Darcy) flow, $K_{n} \rightarrow 0$, then $f \rightarrow 1$ and $K_{a} \rightarrow K$. On the other hand, in the limit of Knudsen diffusion, $K_{n} \rightarrow \infty$, then $\sigma \rightarrow \sigma_{0}$, $f \rightarrow \infty$ and hence $K_{a} \rightarrow K$. Thus in the two extreme limits we have $K_{a} \rightarrow K$.

\section{Numerical Procedure}

The new transport model contains 13 parameters excluding the Forchheimer correction term, and 17 parameters if the Forchheimer correction term is included. All of these parameters must be specified as input to the simulations. With these specified, the correlations for the model parameters and their associated compressibility parameters are completely determined as a function of the local pressure. The system can thus be solved numerically, given appropriate boundary and initial conditions.

We have developed an implicit finite volume solver for the general one-dimensional system. The balance equation 3.38 is discretised and integrated over a typical control volume, to yield a system of nonlinear algebraic equations for the pressure field vector $P_{n}=\left\{p_{1}, p_{2}, \ldots, p_{N}\right\}$, where $p_{i}$ is the pressure at the centre of control volume labeled $i$, at some given time step $n$. Assuming that the system has been solved at time $t=n d t$, then we solve the following system at $t=(n+1) d t$,

$$
A(P) P=S(P)
$$

This is nonlinear algebraic system, so it is solved iteratively by first linearising the matrix of coefficients $A$ and the source term $S$ at the current iteration step, 
i.e. $A^{\nu}=A\left(P^{\nu}\right)$, and $S^{\nu}=S\left(P^{\nu}\right)$. Initially, for $\nu=0$, we have $P^{0}=P_{n}$, so that $A^{0}=A\left(P^{0}\right)$, and $S^{0}=S\left(P^{0}\right)$. Then we solve for $P^{\nu+1}$, until convergence,

$$
\begin{aligned}
& A^{\nu} P^{\nu+1}=S^{\nu}, \\
& P^{\nu+1}=\left(A^{\nu}\right)^{-1} S^{\nu} \\
& \text { define } \epsilon^{\nu+1}=\frac{1}{N} \sum_{i=1}^{N}\left|\frac{p_{i}^{\nu+1}-p_{i}^{\nu}}{p_{i}^{\nu+1}}\right| \\
& \text { if } \epsilon^{\nu+1}<\epsilon_{T o l} \Longrightarrow \text { converged } \\
& \quad \Longrightarrow P_{n+1}=P^{\nu+1} ; \text { go to next time step } n \rightarrow n+1 \\
& \text { else if } \epsilon^{\nu+1}>\epsilon_{T o l} \Longrightarrow \text { not converged } \\
& \Longrightarrow \text { go to next iteration } \nu \rightarrow \nu+1
\end{aligned}
$$

The matrix $A^{\nu}$ is tri-diagonal and can therefore be inverted easily. All the model parameters and compressibility coefficients are fully pressure dependent at all times, and these must be updated at every iteration step $\nu . \epsilon_{T o l}$ is a small tolerance level for the relative error $\epsilon_{\nu}$, typically less than $10^{-4}$.

A flux limiter for large gradients in the pressure field is included. The implicit nature of the solver gives the method good stability.

The solver for a steady-state systems is the same, except that we do not advance the time step.

\section{Determining rock properties using the transport model}

In this work, the main application of the new transport model is to the problem of determining rock properties. This is carried out through solving an inverse problem whereby model parameters are adjusted to fit a given set of experimental data.

Many studies have been conducted to determine the permeability of shale rocks, 


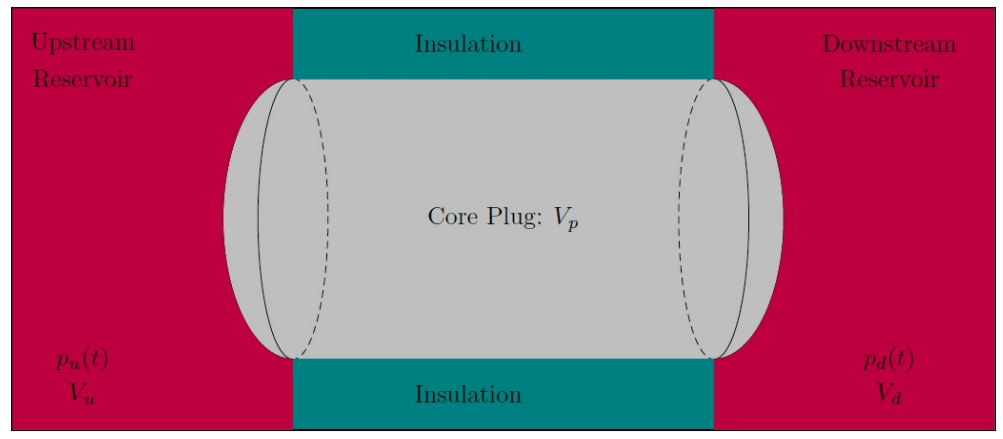

Figure 5: Pressure-pulse decay test setup

but in most of them the reservoir parameters were assumed to be pressure independent or constant in application, see for example Brace et al. [13, Hsieh et al. [40, Neuzil et al. 63, Liang et al. 48, Malkovsky et al. [55, Cui et al. [25, and Civan et al. 222. However, it is more realistic to consider some, if not all, of the model parameters as being pressure dependent, see Pong et al. [67, Beskok and Karniadakis [10, Roy et al. [72, Javadpour et al. [44, Civan et al. [23, Ali et al. 4].

We will use the new transport model equation 3.38 with equations 3.39 and 3.40 in order to estimate rock core properties of rock samples for which we have experimental data from the pressure-pulse decay tests of Pong et al. 67, who used Nitrogen as the working gas. See also, Civan et al. [22], Lorinczi et al. [50, Wang et al. 82, Huang et al. [41, and Ali et al. 44. Pong's data-sets consist of measurements of pressure, $p$, at a number of stations, $x$, along the rock length; this is repeated for several different inlet pressures, $P_{i n}$.

\subsection{Pressure pulse-decay tests}

In a pressure-pulse decay test, Fig. 5, a short rock sample of length $L$ is initially at a constant pressure inside the core sample itself. A pulse of pressure is then sent through the sample from the upstream boundary and the pressure field quickly reaches a steady state distribution across the core length. The pressure 
is recorded at different stations along the core length. Mathematically, we have the following initial condition,

$$
p(x, 0)=p_{o}, \quad 0 \leq x \leq L, \quad t=0,
$$

and Dirichlet Boundary Conditions,

$$
\begin{array}{clrl}
p(0, t) & =p_{u}(t), & x=0, & t>0 . \\
p(L, t) & =p_{d}(t), & x=L, & t>0 .
\end{array}
$$

where $p_{u}$ is the measured values of the pressure in the upstream reservoir, and $p_{d}$ is the measured values of the pressure in the downstream reservoir.

The flux conditions at the inlet (upstream) boundary is $\frac{d\left(\rho V_{u}\right)}{d t}=-\rho \mathbf{u} \cdot \mathbf{n} A$, which for one dimensional domain becomes,

$$
\frac{\partial p}{\partial x}=\left[\frac{V_{u} \mu \phi L}{V_{p}} \frac{\zeta_{\rho}(p)}{F K_{a}}\right] \frac{\partial p}{\partial t}, \quad p=p_{u} ; \quad x=0, \quad t>0 .
$$

where $V_{u}$ is the volume of upstream reservoir, and $A$ is the cross-sectional area.

The flux conditions at the outlet (downstream) boundary is $\frac{d\left(\rho V_{d}\right)}{d t}=\rho \mathbf{u} \cdot \mathbf{n} A$, which for one dimensional domain becomes,

$$
\frac{\partial p}{\partial x}=-\left[\frac{V_{d} \mu \phi L}{V_{p}} \frac{\zeta_{\rho}(p)}{F K_{a}}\right] \frac{\partial p}{\partial t}, \quad p=p_{d} ; \quad x=L, \quad t>0 .
$$

where $V_{d}$ is the volume of downstream reservoir.

In the pressure-pulse decay tests of Pong et al. 67, several different experiments with different inlet pressures $P_{i n}$ were carried out - the values of $P_{i n}$ chosen are listed in Table 1 


$\begin{array}{lc} & \text { Civan's Inflow Conditions } \\ \# & p_{\text {inlet }} \\ 1 & 135 \mathrm{kPa} \\ 2 & 170 \mathrm{kPa} \\ 3 & 205 \mathrm{kPa} \\ 4 & 240 \mathrm{kPa} \\ 5 & 275 \mathrm{kPa}\end{array}$

Table 1: Inflow conditions for Civan's steady state case.

\subsection{Sixteen transport models}

How important is it to keep all parameters to be pressure dependent at all times? To address this question, we consider sixteen different transport models, labeled $k=1,2, \cdots, 16$. These models are produced by taking the four basic compressibility coefficients that appear in the model, $\left(\zeta_{\rho}, \zeta_{K}, \zeta_{f}, \zeta_{\mu}\right)$, to be pressure-dependent or pressure-independent - this gives 16 combinations resulting in the different transport models, listed in Table 2.

Model 1, is when all the parameter are pressure independent, and all compressibility coefficients are zero, $\zeta_{\rho}=\zeta_{K}=\zeta_{f}=\zeta_{\mu}=0$. This collapses to the Darcy law.

Model 16 is the fully pressure dependent case, $\zeta_{\rho} \neq 0, \zeta_{K} \neq 0, \zeta_{f} \neq 0, \zeta_{\mu} \neq 0$.

An initial choice of model parameter values must be made, and these are then adjusted to yield the best-fit choice of model parameters values. The rock properties $K, \phi$, and $\tau$ are determined as the best fit among all the models considered. 


\begin{tabular}{|c|c|c|c|c|c|}
\hline \multirow[b]{2}{*}{ Model \# } & \multicolumn{2}{|c|}{ Compressibility } & \multicolumn{2}{|c|}{ coefficient } & \multirow[b]{2}{*}{ Error } \\
\hline & $\zeta_{\rho}$ & $\zeta_{K}$ & $\zeta_{f}$ & $\zeta_{\mu}$ & \\
\hline 1 & 0 & 0 & 0 & 0 & $2.69 \mathrm{e}-02$ \\
\hline 2 & $p$ & 0 & 0 & 0 & $2.68 \mathrm{e}-02$ \\
\hline 3 & 0 & $p$ & 0 & 0 & $1.64 \mathrm{e}-01$ \\
\hline 4 & 0 & 0 & $p$ & 0 & $1.00 \mathrm{e}+05$ \\
\hline 5 & 0 & 0 & 0 & $p$ & $2.69 \mathrm{e}-02$ \\
\hline 6 & $p$ & $p$ & 0 & 0 & $2.23 \mathrm{e}+00$ \\
\hline 7 & $p$ & 0 & $p$ & 0 & $8.52 \mathrm{e}-01$ \\
\hline 8 & $p$ & 0 & 0 & $p$ & $2.69 \mathrm{e}-02$ \\
\hline 9 & 0 & $p$ & $p$ & 0 & $1.19 \mathrm{e}+00$ \\
\hline 10 & 0 & $p$ & 0 & $p$ & $1.64 \mathrm{e}-01$ \\
\hline 11 & 0 & 0 & $p$ & $p$ & $1.00 \mathrm{e}+05$ \\
\hline 12 & $p$ & $p$ & $p$ & 0 & $1.059 \mathrm{e}-04$ \\
\hline 13 & $p$ & $p$ & 0 & $p$ & $2.23 \mathrm{e}+00$ \\
\hline 14 & $p$ & 0 & $p$ & $p$ & $8.52 \mathrm{e}-01$ \\
\hline 15 & 0 & $p$ & $p$ & $p$ & $1.19 \mathrm{e}+00$ \\
\hline 16 & $p$ & $p$ & $p$ & $p$ & $1.055 \mathrm{e}-04$ \\
\hline
\end{tabular}

Table 2: List of simulations carried out. The four basic compressility coefficients are made pressure-dependent or pressure-independent: in columns 2-5, an entry of ' 0 ' means that the compressibility factor is zero, and an entry of ' $p$ ' means that it is nonzero and the associated physical parameter is function of pressure $p$. Column 6 shows the error from the simulations (with $B=0$ ), using equation (6.8).

\subsection{The general form of $\zeta_{3}(p)$}

From equation (3.24), the most general form of $\zeta_{3}$ in a one-dimensional domain is,

$$
\zeta_{3}(p)=F\left[\zeta_{\rho}(p)+\zeta_{K}(p)+\zeta_{f}(p)-\zeta_{\mu}(p)\right]+(F-1)\left[\zeta_{b}(p)+\zeta_{|u|}(p)\right]
$$

Without the turbulence correction factor, i.e. $B=0$ (or $F=1$ ), we obtain,

$$
\zeta_{3}(p)=\zeta_{\rho}(p)+\zeta_{K}(p)+\zeta_{f}(p)-\zeta_{\mu}(p) .
$$

In the ensuing analysis, we will first consider the simulations from models with- 


\begin{tabular}{ll}
\multicolumn{2}{c}{ Civan (2011) } \\
Reservoir Parameters & Values \\
$L(\mathrm{~m})$ & 0.003 \\
$N_{x}$ & 100 \\
$R_{g}(\mathrm{~J} / \mathrm{kMol} / \mathrm{K})$ & 8314.4 \\
$M_{g}(\mathrm{Kg} / \mathrm{KMol} / \mathrm{K})$ & 28.013 \\
$T(K)$ & 314 \\
$p_{c}(K P a)$ & 3396 \\
$t_{c}(K)$ & 126.19 \\
$b_{S F}$ & -1 \\
$\sigma_{0}$ & 1.3580 \\
$A_{\sigma}$ & 0.1780 \\
$B_{\sigma}$ & 0.4348 \\
$\tau$ & 1 \\
$\zeta_{\tau}\left(P a^{-1}\right)$ & 0 \\
$\phi$ & 0.2 \\
$\zeta_{\phi}\left(P a^{-1}\right)$ & $5 \mathrm{e}-6$ \\
$\mu(P a-s)$ & $1.85 \mathrm{e}-5$ \\
$\zeta_{\mu}\left(P a^{-1}\right)$ & $3 \mathrm{e}-11$ \\
$K$ & $1 \mathrm{e}-15$ \\
$\zeta_{K}\left(P a^{-1}\right)$ & $1 \mathrm{e}-6$
\end{tabular}

Table 3: Reservoir parameters used in Civan's Model (2011).

out Forchheimer's correction term, $B=0(F=1)$, and then the simulations from models with Forchheimer's correction term $B \neq 0(F \neq 1)$.

\subsection{Simulation results}

Many of the model parameters are known either on physical grounds, such as the molecular weight of Nitrogen, or by experimental set-up, such as the size of the domain, $L=3 \mathrm{~mm}$. However, other model parameters and their compressibility coefficients are modeled as described in Sections 3 and 4.

The simulation conditions matched the conditions of Pong's pressure-pulse decay tests with Nitrogen as the working fluid, for five different inlet pressures, $P_{i n}$, shown in Table 1 .

The sixteen transport models were run with the model parameters, listed in Ta- 


\begin{tabular}{ll}
\multicolumn{2}{c}{ New Model } \\
Reservoir Parameters & Values \\
$L(\mathrm{~m})$ & 0.003 \\
$N_{x}$ & 100 \\
$R_{g}(\mathrm{~J} / \mathrm{KMol} / \mathrm{K})$ & 8314.4 \\
$M_{g}(\mathrm{Kg} / \mathrm{KMol} / \mathrm{K})$ & 28.013 \\
$T(\mathrm{~K})$ & 314 \\
$p_{c}(\mathrm{KPa})$ & 3396 \\
$t_{c}(\mathrm{~K})$ & 126.19 \\
$b_{S F}$ & -1 \\
$\sigma_{0}$ & 1.3580 \\
$A_{\sigma}$ & 0.1780 \\
$B_{\sigma}$ & 0.4348 \\
$a_{\tau}$ & $1 \mathrm{e}-6$ \\
$a_{\phi}$ & 0.15 \\
$b_{\phi}$ & $-0.939 \mathrm{e}-06$ \\
$c_{\phi}$ & 2.2 \\
$\alpha_{K C}$ & 1 \\
$\beta_{K C}$ & 0.9 \\
$\Gamma_{K C}$ & $1.9 \mathrm{e}-9$ \\
tol & $1 \mathrm{e}-6$
\end{tabular}

Table 4: Reservoir parameters used in new transport model (??). A data set of base values is obtained which will be used for further data analysis and parameter estimation.

ble 4, which were chosen to match Civan's parameter values, Table 3, where possible. The additional parameters in the new model were initially guesstimated, and then adjusted for best fit. Note that most of Civan's model parameters are made constant.

The relative error between the simulated results, $P^{\text {sim }}$, and the experimental data, $P^{\text {data }}$, is determined from,

$$
\epsilon^{2}=\sum_{i=1}^{N}\left|\frac{p_{i}^{s i m}-p_{i}^{\text {data }}}{p_{i}^{\text {data }}}\right|^{2}
$$

where the summation is taken over the $N$ stations along the $x$-direction where the measurements are recorded; here $N=6$. 


\subsection{Simulation results without Forchheimer's correction $(B=0)$}

The simulation results, without the Forchheimer's correction term, i.e. $B=0$ $(F=1)$, from all sixteen models considered are shown as pressure against the distance along the core sample, in Figures 6(a) - 6(d). The simulation results (lines) are compared with the data of Pong et al. 67. (symbols). The relative errors obtained are shown in Table 2 and plotted in Figure 7.

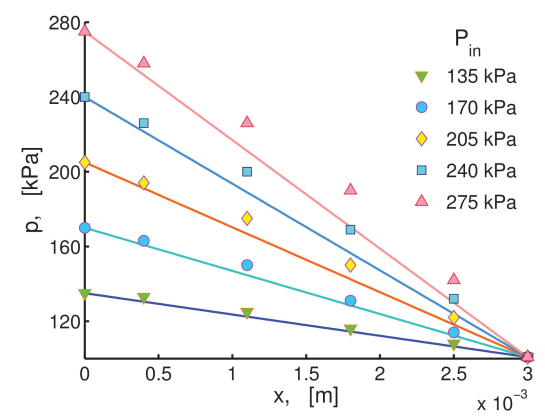

Model 1

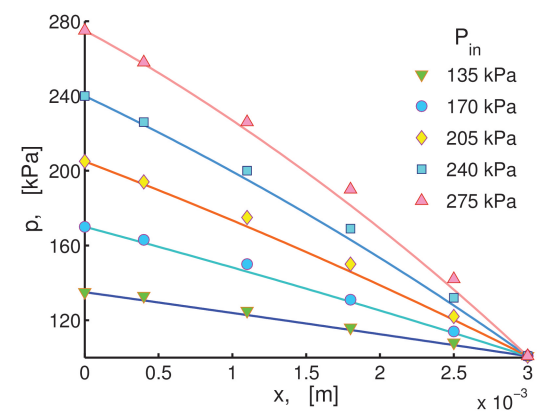

Model 3

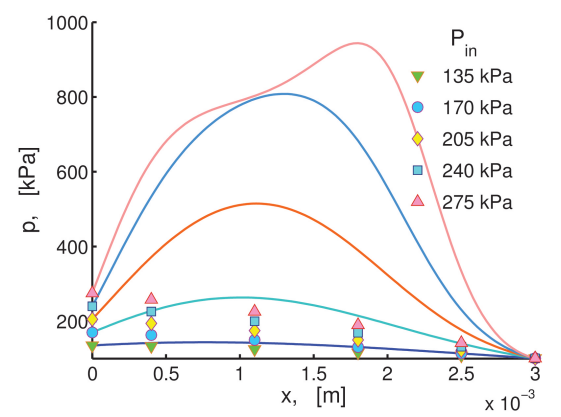

Model 2

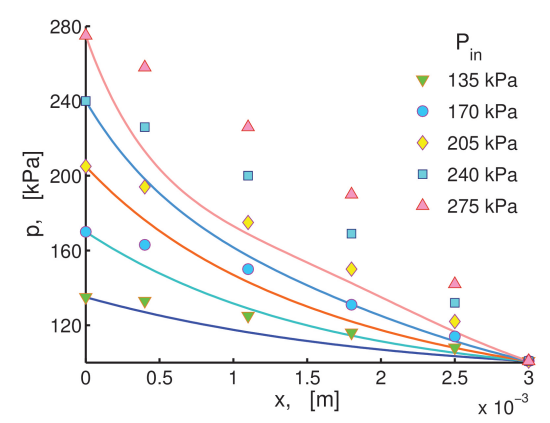

Model 4

Figure 6(a). Simulation results (lines), and experimental data (symbols) from Pong et al. 67, for different inlet flow pressures $P_{i n}$, as indicated. Model numbers and pressure-dependent parameters are shown inTable 2 (Similarly for Figures 6(b)-6(d).)

Only Model 12 and Model 16 show a good match between the numerical solutions and the experimental data. Some of the models show a fairly good match, e.g. Model 3 and Model 10; some of the models show significant errors, e.g. 


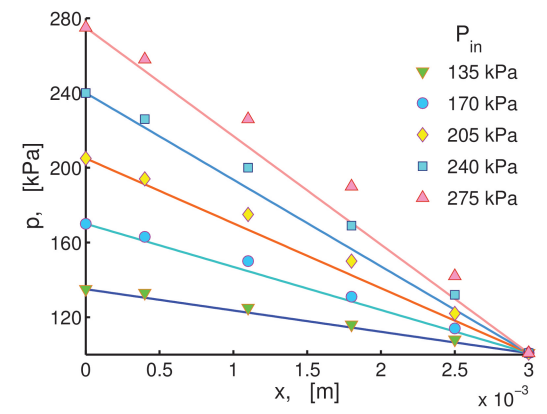

Model 5

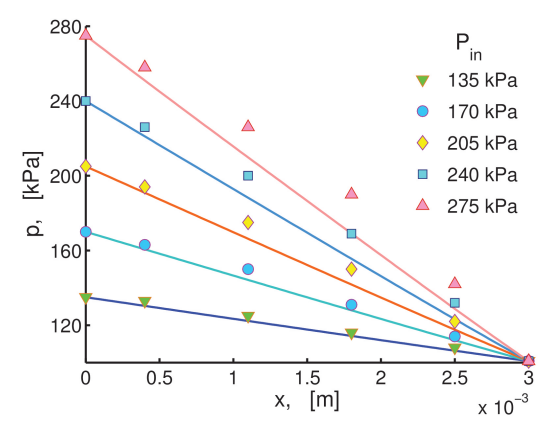

Model 7

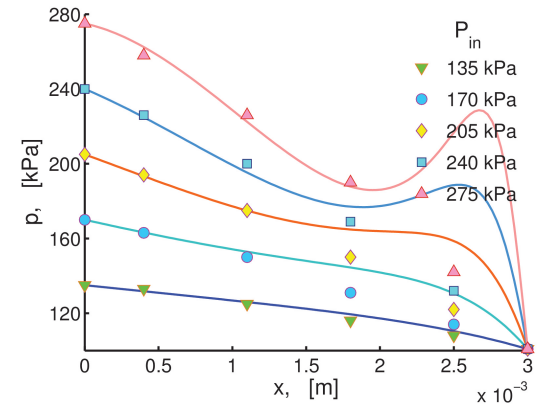

Model 6

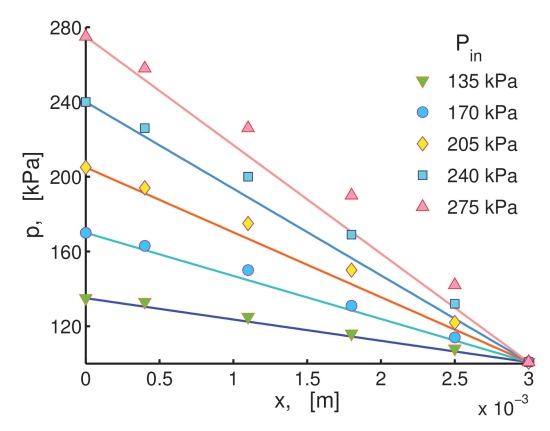

Model 8

Figure 6(b). (See the caption to Figure 6(a)).

Models $1,5,7,8$, and 14; while yet other models are very badly in error, e.g. Models 2, 4, 6, 9, 11, 13, and 15 .

Darcy's law, Model 1, where all model parameters are taken to be independent of the pressure, gives linear profiles and is clearly unsatisfactory.

Fig. 7 show the relative error on log-scale for the sixteen models considered.

Model 16 is the case where all the model parameters are pressure dependent. The error calculated in Model 16 is the smallest among all the sixteen models.

From these results, we conclude that Model 16 is the best fit among all the models considered; this demonstrates the importance of retaining all model parameters to be pressure dependent throughout the simulations in order to 


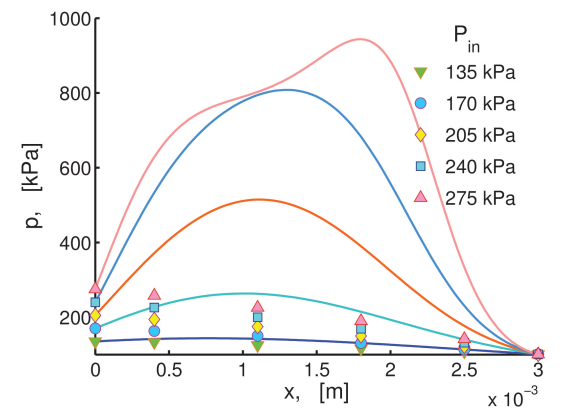

Model 9

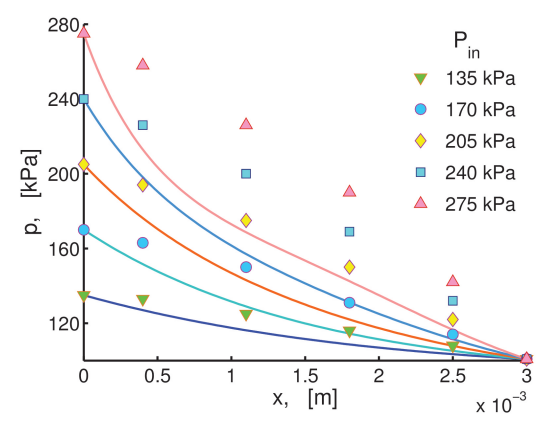

Model 11

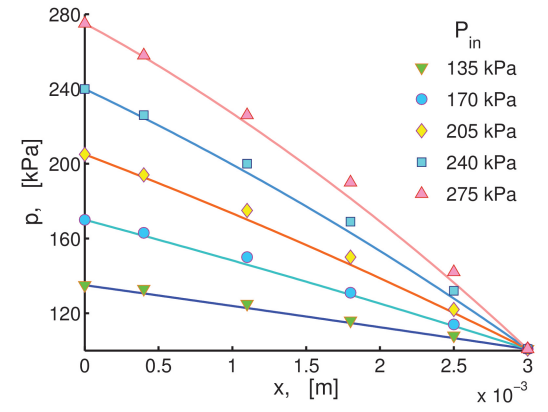

Model 10

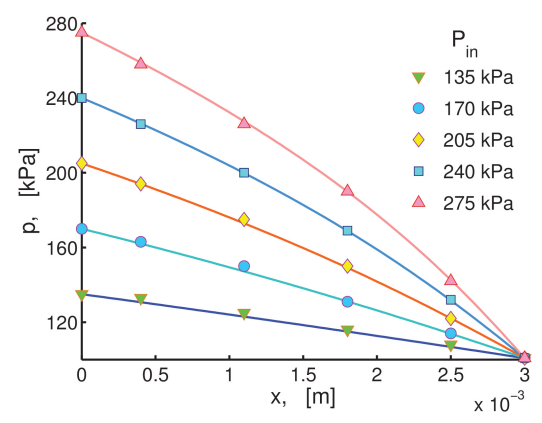

Model 12

Figure 6(c). (See the caption to Figure 6(a)).

obtain the best results.

An exception is for the smallest inlet pressures $P_{i n}$, where most of the models yield fair agreement with the data. This means that low $P_{i n}$ should be avoided in such experiments as the models are not critically sensitive to pressure dependency at low inlet pressures, so estimates of rocks properties cannot be made with accuracy.

From Model 16 we can make estimates of the rock properties. Civan et al. 22] took most of the model parameters to be constant and assumed a constant value for the porosity, $\phi=0.2$, independent of pressure, see Table 3 On this basis his model predicted a value for the rock permeability to be $K=10^{-15} \mathrm{~m}^{2}$, (or $\left.10^{6} \mathrm{nD}\right)$. 


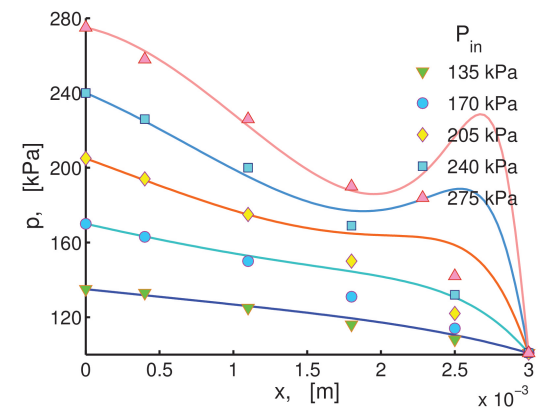

Model 13

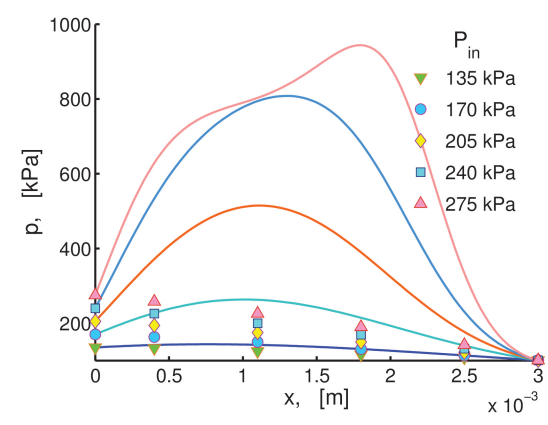

Model 15

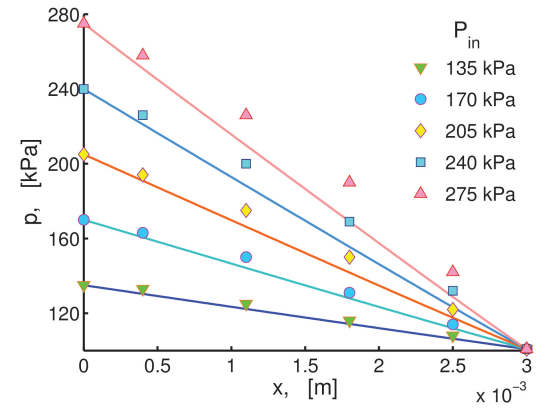

Model 14

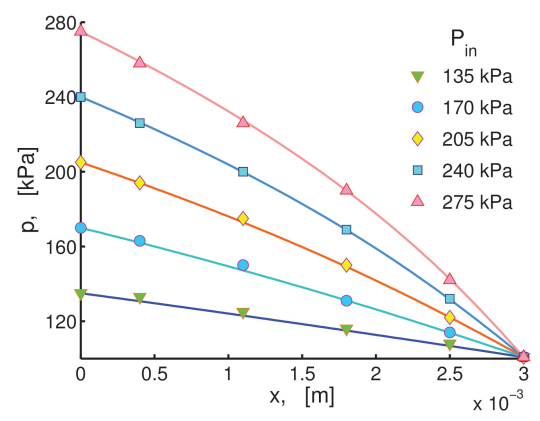

Model 16

Figure 6(d). (See the caption to Figure 6(a)).

In the new model simulations, the porosity is a variable and dependent upon the pressure. From the Model 16 simulations, it lies in the range $0.1901 \leq \phi \leq$ 0.2003 , and it was found that the permeability lies in the range $10^{-14} \leq K \leq$ $10^{-15} \mathrm{~m}^{2}$, (or $10^{6} \leq K \leq 10^{7} \mathrm{nD}$ ). Although these estimates are comparable to Civan's estimate above, these values are much higher than expected, and not realistic of typical shale rocks.

\subsection{Simulation results with Forchheimer's correction, $B \neq 0$}

We now include a non-zero turbulence correction factor $B \neq 0(F \neq 1)$, equation (6.6), to produce a new set of transport models - we will refer to them as Models 1 to 16 with $B \neq 0$. As the critical importance of retaining the pressure- 


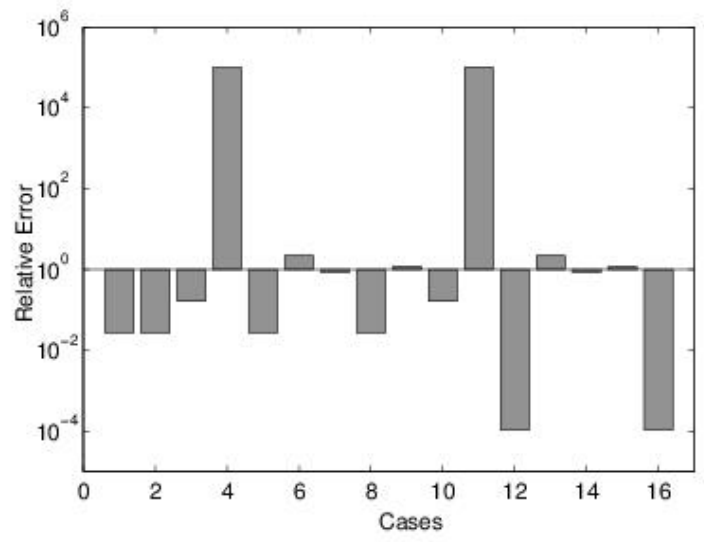

Figure 7. The error, $\epsilon^{2}$ using equation $(6.8)$, between the simulated and experimental data, for the 16 models listed in Table 2 .

dependence of all model parameters has already been established in the previous subsection, here we consider only Model 16, where all parameters are pressuredependent. With $B \neq 0$, we have four additional parameters, $a_{B}, b_{B}, c_{B}$, and $d_{B}$, that arise in the model from the consideration of the Forchheimer's correction term.

Fig. 8 shows the simulation results when we take the same parameter values as for Model 16 with $B=0$, Table 4 . The four additional parameters are, initially, guesstimated. The simulations are significantly in error of the data, except as usual for the lowest $P_{i n}=275 \mathrm{KPa}$.

The model parameters were therefore adjusted for the best fit, and the new list of parameter values is shown in Table 5 . Figure 9 shows the simulation results for different inlet pressures. We observe an excellent match between the numerical solutions and the experimental data. The the relative error between the simulated and the measured pressure values is $5.72 \times 10^{-5}$, which is smaller than from Model 16 with $B=0$, in the previous subsection 6.5, Fig. $6(\mathrm{~d})$.

Importantly, the range of porosity was found to lie in the range $0.10<\phi<$ 0.1038 , and the intrinsic permeability was found to lie in the range $106<K<$ 


\begin{tabular}{|c|c|}
\hline Reservoir Parameters & values \\
\hline$L(\mathrm{~m})$ & 0.003 \\
\hline$N_{x}$ & 100 \\
\hline$R_{g}(\mathrm{JKMol} / \mathrm{K})$ & 8314.4 \\
\hline$M_{g}(\mathrm{Kg} / \mathrm{KMol} / \mathrm{K})$ & 28.013 \\
\hline$T(\mathrm{~K})$ & 314 \\
\hline$p_{c}(\mathrm{KPa})$ & 3396 \\
\hline$t_{c}(\mathrm{~K})$ & 126.19 \\
\hline$b_{S F}$ & -1 \\
\hline$\sigma_{0}$ & 1.3580 \\
\hline$A_{\sigma}$ & 0.1780 \\
\hline$B_{\sigma}$ & 0.4348 \\
\hline$a_{\tau}$ & 1.5 \\
\hline$a_{\phi}$ & 0.10 \\
\hline$b_{\phi}$ & $-0.939 \mathrm{e}-1$ \\
\hline$c_{\phi}$ & 0.39 \\
\hline$\alpha_{K C}$ & 1.0 \\
\hline$\beta_{K C}$ & 0.9 \\
\hline$\Gamma_{K C}$ & $0.72 \mathrm{e}-8$ \\
\hline$a_{\beta}$ & $3.1 \mathrm{e} 01$ \\
\hline$b_{\beta}$ & 0.5 \\
\hline$c_{\beta}$ & 1.35 \\
\hline$d_{\beta}$ & 0.4 \\
\hline Error & $5.972 \mathrm{e}-5$ \\
\hline
\end{tabular}

Table 5: Model parameters used in the New Steady State Model.

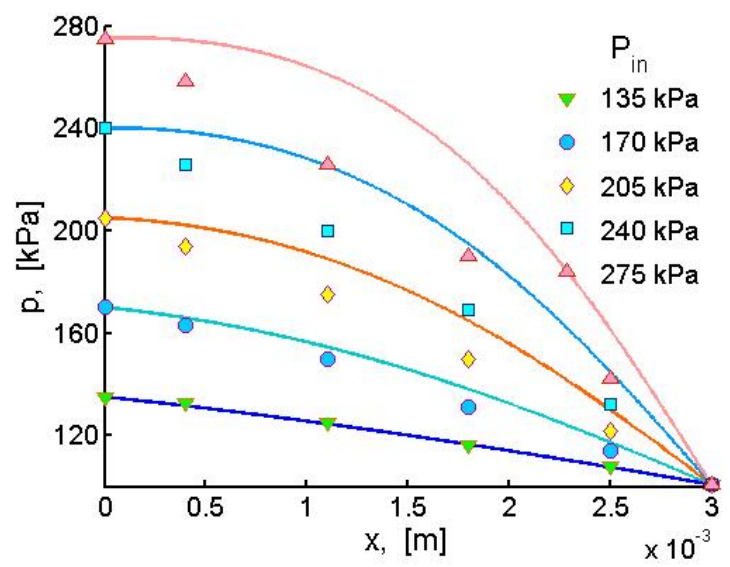

Figure 8. Pressure against distance for different inlet pressures, from Model 16 with $B \neq 0$, and with parameter values listed in Table 4 . 


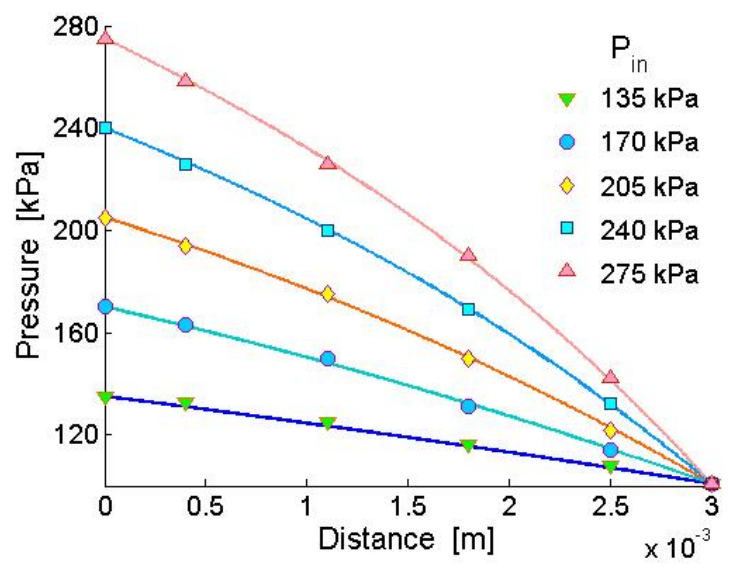

Figure 9. Pressure against distance for different inlet pressures, from Model 16 with $B \neq 0$, and with parameter values listed in Table 5 .

$111 \mathrm{nD}$. These are much more realistic of shale rocks than any previous model.

\section{Discussion and Conclusions}

In this work, a fully pressure-dependent nonlinear transport model for the flow of shale gas in tight porous media has been derived accounting for the important physical processes that exist in the system, such as continuous flow, transition flow, slip flow, surface diffusion, adsorption and desorption in to the rock material, and also including a nonlinear correction term for high flow rates (turbulence). This produces an advection-diffusion type of partial differential equation (PDE) with pressure dependent model parameters and associated compressibility coefficients, and with highly nonlinear apparent convective velocity and apparent diffusivity. The model was developed initially for the general case of transient flow of single phase gas flow in a three-dimensional porous system with gravity and a general source term.

A steady state one-dimensional version of the model without gravity and without external source was used to determine the rock properties by matching the 
pressure distrbution across a shale rock core sample obtained from pressurepulse decay tests for different inflow pressure conditions. It was found that when the high flow rate correction factor is also excluded $(B=0)$, then the model with fully pressure dependent parameters, Model 16, still gives the least errors compared the data, and yields estimates of rock properties that are in close agreement with Civan's model; however, both estimates are not realistic of typical shale rocks.

When the high flow rate correction factor is included $(B \neq 0)$ in the model, the errors are further reduced, and the estimates for the porosity and permeability are much improved and are within the known range of shale rock properties, we believe for the first time. The estimates are more realistic than obtained from previous transport model. This is a noteable achievement for the present model development and application, and sets a benchmark for future development.

We can draw the following conclusions. Firstly, a realistic transport model should incorporate all of the important physical transport sub-processes in the porous system. Secondly, model parameters and associated compressibility coefficients should be pressure dependent throughout the numerical procedure. Thirdly, the a Forchchiemer correction term for high flow rates is very important for good estimation of rock properties. The simulation results presented here for estimating rock properties illustrate the potential of the modelling startegy presented here in producing accurate simulations of gas flow in shale gas reservoirs.

In the future, the second phase of this work is to incorporate this model in to a new model for gas transport through fractured media, such as tight shale rocks.

\section{Acknowledgements}

The authors would like to acknowledge the support provided by King Abdulaziz City for Science and Technology (KACST) through the National Science, 
Technology and Innovation Plan (NSTIP), and through the Science Technology Unit at King Fahd University of Petroleum \& Minerals (KFUPM) for funding this work through project No. 14-OIL280-04.

\section{References}

[1] Aguilera, R., 2010. Flow units: from conventional to tight gas to shale gas reservoirs, in: Trinidad and Tobago Energy Resources Conference.

[2] Aguilera, R., 2014. Flow units: From conventional to tight-gas to shale-gas to tight-oil to shale-oil reservoirs. SPE Reservoir Evaluation \& Engineering 17, 190-208.

[3] Akkutlu, I.Y., Efendlev, Y.,Vasilyeva, Maria 2016. Multiscale model reduction for shale gas transport in fractured media. Comput. Geosci. 20, 953-973.

[4] Ali, I., Chanane, B., Malik, N.A., 2015. Compressibility coeffcients of nonlinear transport models in unconventional gas reservoirs, in: The 2015 AMMCS-CAIMS Congress, Springer. pp. 1-10.

[5] Arthur, J.D., Langhus, B., Alleman, D., 2008. An overview of modern shale gas development in the united states. All Consulting 3, 14-17.

[6] Aybar, U., Eshkalak, M.O., Sepehrnoori, K., Patzek, T.W., 2014. The effect of natural fracture's closure on long-term gas production from unconventional resources. Journal of Natural Gas Science and Engineering 21, 1205-1213.

[7] Aziz, K., Settari, A., 1979. Petroleum reservoir simulation. volume 476. Applied Science Publishers London.

[8] Bear, J., 2013. Dynamics of fluids in porous media. Courier Corporation.

[9] Benzerga, A.A., 2015. Micromechanical models of ductile damage and fracture, in: Handbook of Damage Mechanics. Springer, pp. 939-962. 
[10] Beskok, A., Karniadakis, G.E., 1999. Report: a model for flows in channels, pipes, and ducts at micro and nano scales. Microscale Thermophysical Engineering 3, 43-77.

[11] Bird, G.A., 1983. Definition of mean free path for real gases. Physics of Fluids (1958-1988) 26, 3222-3223.

[12] Bockstiegel, G., 1966. The porosity-pressure curve and its relation to the pore-size distribution in iron powder compacts, in: Modern Developments in Powder Metallurgy. Springer, pp. 155-187.

[13] Brace, W., Walsh, J.B., Frangos, W.T., 1968. Permeability of granite under high pressure. Journal of Geophysical research 73, 2225-2236.

[14] Carman, P.C., Carman, P.C., 1956. Flow of gases through porous media. Butterworths Scientific Publications London.

[15] Chen, L., Zhang, L., Kang, Q., Viswanathan, H.S., Yao, J., Tao, W., 2015. Nanoscale simulation of shale transport properties using the lattice boltzmann method: permeability and diffusivity. Scientific reports 5.

[16] Chen, Z., 2007. Reservoir Simulation: Mathematical Techniques in Oil Recovery. volume 77. SIAM.

[17] Christou, C., Kokou Dadzie, S., 2015. Direct simulation monte carlo method in porous media with varying knudsen number. Society of Petroleum Engineers. doi:10.2118/MS .

[18] Civan, F., 2001. Scale effect on porosity and permeability: Kinetics, model, and correlation. AIChE journal 47, 271-287.

[19] Civan, F., 2010. Effective correlation of apparent gas permeability in tight porous media. Transport in porous media $82,375-384$.

[20] Civan, F., 2011. Porous media transport phenomena. John Wiley \& Sons. 
[21] Civan, F., 2014. Improved permeability prediction for heterogeneous porous media by bundle-of-leaky-tubes with cross-flow model, in: 5th International Conference on Porous Media and Their Applications in Science, Engineering and Industry, ECI Symposium Series. ECI Symposium Series.

[22] Civan, F., Rai, C.S., Sondergeld, C.H., 2011. Shale-gas permeability and diffusivity inferred by improved formulation of relevant retention and transport mechanisms. Transport in Porous Media 86, 925-944.

[23] Civan, F., et al., 2005. Improved permeability equation from the bundle-ofleaky-capillary-tubes model, in: SPE Production Operations Symposium, Society of Petroleum Engineers.

[24] Clarkson, C.R., Solano, N., Bustin, R., Bustin, A., Chalmers, G., He, L., Melnichenko, Y.B., Radliński, A., Blach, T.P., 2013. Pore structure characterization of north american shale gas reservoirs using usans/sans, gas adsorption, and mercury intrusion. Fuel 103, 606-616.

[25] Cui, X., Bustin, A.M.M., Bustin, R.M., 2009. Measurements of gas permeability and diffusivity of tight reservoir rocks: different approaches and their applications. Geofluids 9, 208-223.

[26] Cussler, E.L., 2009. Diffusion: mass transfer in fluid systems. Cambridge university press.

[27] Darcy, H., 1856. Les Fontaines publiques de la Ville de Dijon. Dalmount, Paris.

[28] Darishchev, A., Rouvroy, P., Lemouzy, P., 2013. On simulation of flow in tight and shale gas reservoirs, in: 2013 SPE Middle East Unconventional Gas Conference \& Exhibition.

[29] Estrada, J.M., Bhamidimarri, R., 2016. A review of the issues and treatment options for wastewater from shale gas extraction by hydraulic fracturing. Fuel 182, 292-303. 
[30] Evans, R.D., Civan, F., 1994. Characterization of non-Darcy multiphase flow in petroleum bearing formations. Report, US DOE Contract No. Technical Report. DE-AC22-90BC14659, School of Petroleum and Geological Engineering, University of Oklahoma.

[31] Faybishenko, B., Benson, S.M., Gale, J.E., Molz, F., 2015. A complex systems approach to describing flow and transport in fractured-porous media. Fluid Dynamics in Complex Fractured-Porous Systems 7, 5.

[32] Fernandes, B.R.B., Varavei, A., Marcondes, F., Sepehrnoori, K., 2014. Comparison of an impec and a semi-implicit formulation for compositional reservoir simulation. Brazilian Journal of Chemical Engineering 31, 977991.

[33] Foo, K.Y., Hameed, B.H., 2010. Insights into the modeling of adsorption isotherm systems. Chemical Engineering Journal 156, 2-10.

[34] Forchheimer, P., 1901. Wasserbewegung durch boden. Z. Ver. Deut. Ing. $45,1782-1788$.

[35] Freeman, C.M., Moridis, G.J., Blasingame, T.A., 2011. A numerical study of microscale flow behavior in tight gas and shale gas reservoir systems. Transport in porous media 90, 253-268.

[36] Geng, L., Li, G., Zitha, P., Tian, S., Sheng, M., Fan, X., 2016. A diffusionviscous flow model for simulating shale gas transport in nano-pores. Fuel 181, 887-894.

[37] Guo, C., Xu, J., Wei, M., Jiang, R., 2015a. Experimental study and numerical simulation of hydraulic fracturing tight sandstone reservoirs. Fuel $159,334-344$.

[38] Guo, C., Xu, J., Wu, K., Wei, M., Liu, S., 2015b. Study on gas flow through nano pores of shale gas reservoirs. Fuel 143, 107-117. 
[39] Hanea, R., Evensen, G., Hustoft, L., Ek, T., Chitu, A., Wilschut, F., et al., 2015. Reservoir management under geological uncertainty using fast model update, in: SPE Reservoir Simulation Symposium, Society of Petroleum Engineers.

[40] Hsieh, P.A., Tracy, J.V., Neuzil, C.E., Bredehoeft, J.D., Silliman, S.E., 1981. A transient laboratory method for determining the hydraulic properties of tight rocks i. theory, in: International Journal of Rock Mechanics and Mining Sciences \& Geomechanics Abstracts, Elsevier. pp. 245-252.

[41] Huang, C., Matsuda, Y., Gregory, J.W., Nagai, H., Asai, K., 2015. The applications of pressure-sensitive paint in microfluidic systems. Microfluidics and Nanofluidics 18, 739-753.

[42] Huang, H., Ayoub, J.A., et al., 2008. Applicability of the forchheimer equation for non-darcy flow in porous media. SPE Journal 13, 112-122.

[43] Islam, M.R., . Unconventional Gas Reservoirs: Evaluation, Appraisal, and Development.

[44] Javadpour, F., et al., 2009. Nanopores and apparent permeability of gas flow in mudrocks (shales and siltstone). Journal of Canadian Petroleum Technology 48, 16-21.

[45] Jones, F.O., Owens, W.W., et al., 1980. A laboratory study of lowpermeability gas sands. Journal of Petroleum Technology 32, 1-631.

[46] Klinkenberg, L.J., et al., 1941. The permeability of porous media to liquids and gases, in: Drilling and production practice, American Petroleum Institute.

[47] Li, D., Engler, T.W., et al., 2001. Literature review on correlations of the non-darcy coefficient, in: SPE Permian Basin Oil and Gas Recovery Conference, Society of Petroleum Engineers. 
[48] Liang, Y., Price, J.D., Wark, D.A., Watson, E.B., 2001. Nonlinear pressure diffusion in a porous medium: Approximate solutions with applications to permeability measurements using transient pulse decay method. Journal of Geophysical Research: Solid Earth (1978-2012) 106, 529-535.

[49] Loeb, L.B., 2004. The kinetic theory of gases. Courier Dover Publications.

[50] Lorinczi, P., Burns, A.D., Lesnic, D., Fisher, Q.J., Crook, A.J., Grattoni, C., Rybalcenko, K., 2014. Direct and inverse methods for determining gas flow properties of shale, in: SPE/EAGE European Unconventional Resources Conference and Exhibition.

[51] Ma, J., Sanchez, J.P., Wu, K., Couples, G.D., Jiang, Z., 2014. A pore network model for simulating non-ideal gas flow in micro-and nano-porous materials. Fuel 116, 498-508.

[52] Macini, P., Mesini, E., Viola, R., 2011. Laboratory measurements of nondarcy flow coefficients in natural and artificial unconsolidated porous media. Journal of Petroleum Science and Engineering 77, 365-374.

[53] Mahdi, R.A., Mohammed, H.A., Munisamy, K.M., Saeid, N.H., 2015. Review of convection heat transfer and fluid flow in porous media with nanofluid. Renewable and Sustainable Energy Reviews 41, 715-734.

[54] Mahmoud, M., 2014. Development of a new correlation of gas compressibility factor (z-factor) for high pressure gas reservoirs. Journal of Energy Resources Technology 136, 012903.

[55] Malkovsky, V.I., Zharikov, A.V., Shmonov, V.M., 2009. New methods for measuring the permeability of rock samples for a single-phase fluid. Izvestiya, Physics of the Solid Earth 45, 89-100.

[56] Marcondes, F., Santos, L.S., Varavei, A., Sepehrnoori, K., 2013. A 3d hybrid element-based finite-volume method for heterogeneous and anisotropic compositional reservoir simulation. Journal of Petroleum Science and Engineering 108, 342-351. 
[57] Marcondes, F., Sepehrnoori, K., 2010. An element-based finite-volume method approach for heterogeneous and anisotropic compositional reservoir simulation. Journal of Petroleum Science and Engineering 73, 99-106.

[58] Matyka, M., Khalili, A., Koza, Z., 2008. Tortuosity-porosity relation in porous media flow. Physical Review E 78, 026306.

[59] Moghaddam, R.N., Jamiolahmady, M., 2016. Slip flow in porous media. Fuel 173, 298-310.

[60] Møyner, O., Krogstad, S., Lie, K.A., et al., 2015. The application of flow diagnostics for reservoir management. SPE Journal 20, 306-323.

[61] Muljadi, B.P., Blunt, M.J., Raeini, A.Q., Bijeljic, B., 2015. The impact of porous media heterogeneity on non-darcy flow behaviour from pore-scale simulation. Advances in Water Resources .

[62] Müller-Huber, E., Schön, J., Börner, F., 2015. The effect of a variable pore radius on formation resistivity factor. Journal of Applied Geophysics 116, $173-179$.

[63] Neuzil, C.E., Cooley, C., Silliman, S.E., Bredehoeft, J.D., Hsieh, P.A., 1981. A transient laboratory method for determining the hydraulic properties of tight rocks ii. application, in: International Journal of Rock Mechanics and Mining Sciences \& Geomechanics Abstracts, Elsevier. pp. 253-258.

[64] Nia, S., Dasani, D., Tsotsis, T., Jessen, K., 2013. Pore-scale characterization of oil-rich monterey shale: A preliminary study, in: Unconventional Resources Technology Conference.

[65] Oliver, D., Chen, Y., 2011. Recent progress on reservoir history matching: a review. Comput. Geosci. 15, 185-221.

[66] Peaceman, D.W., 2000. Fundamentals of numerical reservoir simulation. Elsevier, New York. 
[67] Pong, K., Ho, C., Liu, J., Tai, Y., 1994. Non-linear pressure distribution in uniform microchannels. ASME Fluids Engineering Division (FED) 197, $51-56$.

[68] Prada, A., Civan, F., 1999. Modification of darcy's law for the threshold pressure gradient. Journal of Petroleum Science and Engineering 22, 237240.

[69] Ramakrishnan, T.S., Goode, P.A., 2015. Measurement of off-diagonal transport coefficients in two-phase flow in porous media. Journal of colloid and interface science 449, 392-398.

[70] Rathakrishnan, E., 2013. Gas Dynamics. PHI Learning Pvt. Ltd., New Delhi.

[71] Regnet, J.B., David, C., Fortin, J., Robion, P., Makhloufi, Y., Collin, P.Y., 2015. Influence of microporosity distribution on the mechanical behavior of oolithic carbonate rocks. Geomechanics for Energy and the Environment $3,11-23$.

[72] Roy, S., Raju, R., Chuang, H.F., Cruden, B.A., Meyyappan, M., 2003. Modeling gas flow through microchannels and nanopores. Journal of applied physics $93,4870-4879$.

[73] Satter, A., Varnon, J.E., Hoang, M.T., et al., 1994. Integrated reservoir management. Journal of Petroleum Technology 46, 1-057.

[74] Soeder, D.J., 2012. Shale gas development in the United States. INTECH Open Access Publisher.

[75] Song, W., Yao, J., Li, Y., Sun, H., Zhang, L., Yang, Y., Zhao, J., Sui, H., 2016. Apparent gas permeability in an organic-rich shale reservoir. Fuel 181, 973-984.

[76] Su, Y., Davidson, J.H., 2015. A uniform theoretical model for fluid flow and heat transfer in porous media, in: Modeling Approaches to Natural Convection in Porous Media. Springer, pp. 9-15. 
[77] Sun, H., Yao, J., Fan, D., Wang, C., Sun, Z., 2015. Gas transport mode criteria in ultra-tight porous media. International Journal of Heat and Mass Transfer 83, 192-199.

[78] Thauvin, F., Mohanty, K.K., 1998. Network modeling of non-darcy flow through porous media. Transport in Porous Media 31, 19-37.

[79] Vafai, K., 2005. Handbook of porous media. Crc Press.

[80] Vesters, J.B., Zitha, P.L.J., Blandamour, E.H., 2013. An improved method for the monitoring of the productivity of .

[81] Walsh, J.B., Brace, W.F., 1984. The effect of pressure on porosity and the transport properties of rock. Journal of Geophysical Research: Solid Earth (1978-2012) 89, 9425-9431.

[82] Wang, J., Luo, H., Liu, H., Ji, Y., Cao, F., Li, Z., Sepehrnoori, K., et al., 2015. Variations of gas flow regimes and petro-physical properties during gas production considering volume consumed by adsorbed gas and stress dependence effect in shale gas reservoirs, in: SPE Annual Technical Conference and Exhibition, Society of Petroleum Engineers.

[83] Wang, Q., Chen, X., Jha, A.N., Rogers, H., 2014. Natural gas from shale formation-the evolution, evidences and challenges of shale gas revolution in united states. Renewable and Sustainable Energy Reviews 30, 1-28.

[84] Wang, Y., Shahvali, M., 2016. Discrete fracture modeling using centroidal voronoi grid for simulation of shale gas plays with coupled nonlinear physics. Fuel 163, 65-73.

[85] Wang, Y., Yan, B., Killough, J., 2013. Compositional modeling of tight oil using dynamic nanopore properties, in: SPE Annual Technical Conference and Exhibition.

[86] Xu, J., Jiang, R., Fu, J., Jiang, Y., 2015. A new numerical simulation method for horizontal well in tight sandstone reservoirs. Journal of Petroleum Science Research . 
[87] Xu, P., Yu, B., 2008. Developing a new form of permeability and kozenycarman constant for homogeneous porous media by means of fractal geometry. Advances in water resources $31,74-81$.

[88] Zhang, A., 2013. Numerical investigation of multiphase Darcy-Forchheimer flow and contaminant transport during $\mathrm{SO}_{2}$ co-injection with $\mathrm{CO}_{2}$ in deep saline aquifers. Thesis. Georgia Institute of Technology.

[89] Zhang, W., Yao, J., Gao, Y., Zhang, Q., Sun, H., 2015. Analysis of electrokinetic coupling of fluid flow in porous media using a 3-d pore network. Journal of Petroleum Science and Engineering 134, 150-157.

[90] Zheng, J., Zheng, L., Liu, H., Ju, Y., 2015. Relationships between permeability, porosity and effective stress for low-permeability sedimentary rock. International Journal of Rock Mechanics and Mining Sciences 78, 304-318.

[91] Ziarani, A.S., Aguilera, R., 2012. Knudsen's permeability correction for tight porous media. Transport in porous media 91, 239-260. 